\title{
Lspm J1112+7626: Detection of a 41 Day M-dwarf Eclipsing Binary From the Mearth Transit Survey
}

\section{Citation}

Irwin, Jonathan M., Samuel N. Quinn, Zachory K. Berta, David W. Latham, Guillermo Torres, Christopher J. Burke, David Charbonneau, et al. 2011. "LSPM J1112+7626: DETECTION OF A 41 DAY M-DWARF ECLIPSING BINARY FROM THE MEARTH TRANSIT SURVEY." The Astrophysical Journal 742 (2): 123. https://doi.org/10.1088/0004-637x/742/2/123.

\section{Permanent link}

http://nrs.harvard.edu/urn-3:HUL.InstRepos:41397455

\section{Terms of Use}

This article was downloaded from Harvard University's DASH repository, and is made available under the terms and conditions applicable to Other Posted Material, as set forth at http:// nrs.harvard.edu/urn-3:HUL.InstRepos:dash.current.terms-of-use\#LAA

\section{Share Your Story}

The Harvard community has made this article openly available. Please share how this access benefits you. Submit a story.

Accessibility 


\title{
LSPM J1112+7626: DETECTION OF A 41 DAY M-DWARF ECLIPSING BINARY FROM THE MEARTH TRANSIT SURVEY
}

\author{
Jonathan M. Irwin ${ }^{1}$, SAmuel N. QuinN ${ }^{1}$, Zachory K. Berta ${ }^{1}$, David W. Latham ${ }^{1}$, Guillermo Torres ${ }^{1}$, \\ Christopher J. Burke ${ }^{1}$, David Charbonneau ${ }^{1}$, Jason Dittmann ${ }^{1}$, Gilbert A. Esquerdo ${ }^{1}$, Robert P. Stefanik ${ }^{1}$, \\ Arto Oksanen $^{2}$, Lars A. Buchhave ${ }^{3,4}$, Philip Nutzman ${ }^{5}$, Perry Berlind ${ }^{6}$, Michael L. Calkins ${ }^{6}$, and Emilio E. Falco ${ }^{6}$ \\ ${ }^{1}$ Harvard-Smithsonian Center for Astrophysics, 60 Garden St., Cambridge, MA 02138, USA; jirwin@ cfa.harvard.edu \\ ${ }^{2}$ Hankasalmi Observatory, Jyväskylän Sirius ry, Vertaalantie 419, FI-40270 Palokka, Finland \\ ${ }^{3}$ Niels Bohr Institute, University of Copenhagen, DK-2100 Copenhagen, Denmark \\ ${ }^{4}$ Centre for Star and Planet Formation, Natural History Museum of Denmark, University of Copenhagen, DK-1350 Copenhagen, Denmark \\ ${ }^{5}$ Department of Astronomy and Astrophysics, University of California, Santa Cruz, CA 95064, USA \\ ${ }^{6}$ Fred Lawrence Whipple Observatory, Smithsonian Astrophysical Observatory, 670 Mount Hopkins Road, Amado, AZ 85645, USA \\ Received 2011 August 8; accepted 2011 September 8; published 2011 November 15
}

\begin{abstract}
We report the detection of eclipses in LSPM J1112+7626, which we find to be a moderately bright $\left(I_{C}=\right.$ $12.14 \pm 0.05)$ very low mass binary system with an orbital period of $41.03236 \pm 0.00002$ days, and component masses $M_{1}=0.395 \pm 0.002 M_{\odot}$ and $M_{2}=0.275 \pm 0.001 M_{\odot}$ in an eccentric $(e=0.239 \pm 0.002)$ orbit. A 65 day out-of-eclipse modulation of approximately $2 \%$ peak-to-peak amplitude is seen in $I$-band, which is probably due to rotational modulation of photospheric spots on one of the binary components. This paper presents the discovery and characterization of the object, including radial velocities sufficient to determine both component masses to better than $1 \%$ precision, and a photometric solution. We find that the sum of the component radii, which is much better determined than the individual radii, is inflated by $3.8_{-0.5}^{+0.9} \%$ compared to the theoretical model predictions, depending on the age and metallicity assumed. These results demonstrate that the difficulties in reproducing observed M-dwarf eclipsing binary radii with theoretical models are not confined to systems with very short orbital periods. This object promises to be a fruitful testing ground for the hypothesized link between inflated radii in M-dwarfs and activity.
\end{abstract}

Key words: binaries: eclipsing - stars: low-mass

Online-only material: color figures, machine-readable table

\section{INTRODUCTION}

Detached, double-lined eclipsing binaries (EBs) provide a largely model-independent means to precisely and accurately measure fundamental stellar properties, particularly masses and radii. In the best-observed systems the precision of these can be at the $<1 \%$ level, and thus place stringent constraints on stellar evolution models (e.g., Andersen 1991; Torres et al. 2010).

Despite the ubiquity of M-dwarfs in the solar neighborhood, their fundamental properties are poorly understood. This is particularly the case below $0.35 M_{\odot}$, the boundary at which field stars are thought to become fully convective (e.g., Chabrier \& Baraffe 1997). Furthermore, observations of many of the bestcharacterized M-dwarf EBs indicate significant discrepancies with the stellar models.

The components of CM Dra, the best-characterized doublelined system in the fully convective mass range (Eggen \& Sandage 1967; Lacy 1977; Metcalfe et al. 1996; Morales et al. 2009), have radii approximately $5 \%-7 \%$ larger than predicted by theoretical stellar evolution models. The observation of inflated radii for M-dwarf binaries at the 5\%-10\% level, and effective temperatures $3 \%-5 \%$ lower than the models predict, is nearly ubiquitous among the best-characterized objects (e.g., Torres et al. 2010, and references therein; Morales et al. 2010; Kraus et al. 2011), although it is interesting to note that Carter et al. (2011) find a smaller inflation for KOI-126B and C, members of a fascinating system containing a short-period M-dwarf binary orbiting a K star, where the system undergoes mutual eclipses allowing a precise determination of the radii of both M-dwarfs.
Two main hypotheses have been advanced to explain the inflated radii in low-mass EBs: metallicity (Berger et al. 2006), and the effect of magnetic activity (the "activity hypothesis," e.g., López-Morales \& Ribas 2005; Ribas 2006; Chabrier et al. 2007).

As discussed by López-Morales (2007), one of the main difficulties with metallicity as an explanation is that current models of M-dwarfs yield radii differing only by a very small amount (an increase of approximately 3\%) between $[\mathrm{M} / \mathrm{H}]=-0.5-0$, the range spanned by the available Baraffe et al. (1998) models. Unless the EB sample contains many objects of very high metallicity, this effect does not seem large enough. $^{7}$

Observationally, the difficulty of determining metallicities for M-dwarfs (e.g., Bonfils et al. 2005; Woolf \& Wallerstein 2006; Bean et al. 2006) renders the metallicity hypothesis difficult to test in practice, and this is further exacerbated by the double lines and rapid rotation in most M-dwarf EB spectra. ${ }^{8}$ Nonetheless, it is interesting to note that $\mathrm{CM}$ Dra is thought to be metal poor (Viti et al. 1997, 2002), which would increase the size of the radius discrepancy for this object, rather than decreasing it, and more generally, this is expected to also be true of other EBs in the sample, assuming they follow the metallicity distribution of field stars (e.g., Carney et al. 1989).

\footnotetext{
7 As discussed by López-Morales (2007), it is possible the effect on the radius is suppressed by a missing source of opacity in the models. Such effects are known to exist as the same models fail to reproduce the observed optical spectra and colors of M-dwarfs (Baraffe et al. 1998).

8 We thank the referee for bringing this point to our attention.
} 
Given these lines of reasoning, it seems unlikely that metallicity is the only explanation for the inflated radii (although it probably plays a role, particularly in creating scatter in the effective temperatures). This has led to the "activity hypothesis" becoming the preferred explanation in the literature for the inflation. It is motivated by noting that nearly all EB systems have short orbital periods. For example, below $0.35 M_{\odot}$, the

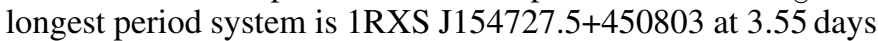
(Hartman et al. 2011). At such short periods, the components are expected to have been tidally synchronized and the orbits circularized by field ages. The effect of the companion and tidal locking is likely to significantly increase the activity levels. The available observational evidence supports this, with the EBs below $0.35 M_{\odot}$ all showing signs of high activity and tidal effects including synchronous, rapid rotation, large amplitude out-ofeclipse modulations, $\mathrm{H} \alpha$ emission, $\mathrm{X}$-ray emission, and in most cases, circular orbits.

Magnetic activity could have an effect either by inhibiting convection (Mullan \& MacDonald 2001; Chabrier et al. 2007) or due to reduced heat flux resulting from cool photospheric spots. Of these, Chabrier et al. (2007) find that the structure of objects below $0.35 M_{\odot}$ is almost independent of variations in the mixing length parameter, which they use to implement inhibition of convection, so the latter possibility (inhibition of radiation due to the effect of spots) seems more likely the explanation in the mass domain of particular interest here. It is important to note that spots also influence the solution of EB light curves. The possibility of such systematic errors in the observations is discussed by Morales et al. (2010), and we shall return to it later in the analysis.

An obvious way to test the activity hypothesis is to measure radii for components of EBs with long orbital periods, where tides are unimportant, and the stars should rotate close to the rates expected for single stars in the absence of a binary companion. Unfortunately, such an endeavor is difficult due to the reduced geometric alignment probability and limited availability of long-duration photometric monitoring of $\mathrm{M}$ dwarfs at rapid cadences, where the majority of wide-field variability surveys are carried out in visible bandpasses, often yielding poor signal-to-noise ratios for M-dwarfs, which are faint at these wavelengths.

The longest period M-dwarf EB with a published orbit is T-Lyr1-17236 (Devor et al. 2008), which has an 8.4 day orbital period and components of 0.68 and $0.52 M_{\odot}$. The radii are indeed consistent with the theoretical models, but the observational uncertainties are still quite large in the discovery paper. This object is currently being observed by the NASA Kepler mission, so a more precise solution should be forthcoming.

In light of the difficulty of identifying longer-period EBs, several authors have attempted to test the activity hypothesis using the existing EB sample to search for correlations between, e.g., orbital period or activity measures and radius inflation (López-Morales 2007; Kraus et al. 2011). While there does appear to be evidence for such a correlation above $0.35 M_{\odot}$, below this mass the present sample is limited by small number statistics and the narrow range of orbital periods spanned. The Kepler mission appears to have identified a large number of very long period EB systems (Prša et al. 2011; see also Coughlin et al. 2011 where more detailed solutions using the Kepler photometry are presented for low-mass objects from this sample), but we are not aware of published dynamical masses and modelindependent radii for these at the present time, and many of the M-dwarfs (especially below $0.35 M_{\odot}$ ) are secondaries in systems with mass ratios much less than unity, where detecting the secondary spectrum to measure radial velocities may be challenging.

An alternative method to determine M-dwarf radii is by making interferometric measurements of angular diameters for (usually isolated) stars. Combined with trigonometric parallaxes and minor assumptions about the stellar limb darkening, the physical radius of the star can be inferred in a model-independent fashion. The difficulty with this method comes in estimating the stellar mass. Dynamical measurements are usually not available, so one has to resort to inferring mass from other measurable stellar properties, necessarily with larger uncertainties. Typically, absolute magnitude in near-infrared passbands is used, which is found to correlate well with stellar mass and has a relatively small scatter. The empirical polynomials of Delfosse et al. (2000) are the most widely used, although there is some argument in the literature as to how accurately these predict the mass, especially over a range of metallicity and spectral type (the uncertainties are probably $\geqslant 10 \%$ ). These concerns combined with small sample sizes mean interferometry results have thus far remained somewhat ambiguous (e.g., Demory et al. 2009; Boyajian et al. 2011, and references therein).

In this contribution, we present the discovery of eclipses in LSPM J1112+7626, a nearby double-lined M-dwarf system with an extremely long orbital period (compared to existing well-characterized M-dwarf EBs) of approximately 41 days. At this large orbital separation the system is not expected to have experienced significant tidal effects (e.g., Zahn 1977) and is predicted to be neither synchronized nor circularized, as verified by the non-circular orbit and apparent non-synchronized spin and orbit for one of the components as we shall describe. In the absence of strong tidal effects, it is expected that the angular momentum evolution of each component has not been perturbed significantly by the binary companion, and therefore the stars should possess magnetic properties more representative of single M-dwarfs. In this contribution, we proceed to derive a high-quality spectroscopic orbit and a photometric solution for the system.

\section{OBSERVATIONS AND DATA REDUCTION}

\subsection{Initial Detection}

LSPM J1112+7626 was targeted as part of routine operation of the MEarth transit survey (Nutzman \& Charbonneau 2008; Irwin et al. 2009a) during the 2009-2010 season, with the first observations taken on UT 2009 December 2. Exposure times for the LSPM J1112+7626 field were $27 \mathrm{~s}$, taking three exposures per visit (the total exposure times are tailored for each target to achieve sensitivity to a particular planet size for the assumed stellar radius), with visits at a cadence of 20 minutes.

Eclipses were first detected in LSPM J1112+7626 on UT 2010 April 3 (this was a primary eclipse), and subsequently confirmed on UT 2010 April 28 by observing the majority of a secondary eclipse egress. Both eclipses were detected by an experimental automated real-time eclipse detection and followup system we began to trial during the 2009-2010 season (this will be described in detail in a future publication when it has matured). Confident that the object was an EB, but still with an ambiguous orbital period, we immediately commenced radial velocity monitoring. The radial velocities ultimately determined which of the possible orbital periods was correct, in conjunction 
Table 1

Light Curve Data

\begin{tabular}{|c|c|c|c|c|c|c|c|c|c|c|c|}
\hline $\mathrm{ID}^{\mathrm{a}}$ & Orbit $^{b}$ & $\begin{array}{c}\text { HJD_UTC } \\
\text { (days) }\end{array}$ & $\begin{array}{c}I \\
(\mathrm{mag})\end{array}$ & $\begin{array}{c}\sigma(I) \\
(\mathrm{mag})\end{array}$ & $\begin{array}{r}\Delta \mathrm{mag}^{\mathrm{c}} \\
(\mathrm{mag})\end{array}$ & $\begin{array}{c}\mathrm{FWHM}^{\mathrm{d}} \\
\text { (pix) }\end{array}$ & Airmass & MeridFlag $^{\mathrm{e}}$ & $\begin{array}{c}x \\
\text { (pix) }\end{array}$ & $\begin{array}{c}y \\
\text { (pix) }\end{array}$ & $\begin{array}{l}\mathrm{CM}^{\mathrm{f}} \\
(\mathrm{mag})\end{array}$ \\
\hline $\mathrm{C}$ & 1 & 2455355.573517 & -0.0224 & 0.0073 & 0.000 & 2.10 & 1.2814 & 0 & 100.427 & 378.595 & 0.0000 \\
\hline $\mathrm{C}$ & 1 & 2455355.574050 & -0.0168 & 0.0077 & -0.002 & 2.19 & 1.2820 & 0 & 100.715 & 378.567 & 0.0000 \\
\hline $\mathrm{C}$ & 1 & 2455355.574547 & -0.0161 & 0.0079 & -0.000 & 2.18 & 1.2827 & 0 & 100.936 & 378.442 & 0.0000 \\
\hline $\mathrm{C}$ & 1 & 2455355.575091 & -0.0176 & 0.0079 & -0.033 & 2.26 & 1.2833 & 0 & 100.904 & 378.255 & 0.0000 \\
\hline $\mathrm{C}$ & 1 & 2455355.575624 & -0.0165 & 0.0081 & -0.010 & 2.20 & 1.2840 & 0 & 100.900 & 378.023 & 0.0000 \\
\hline
\end{tabular}

Notes.

${ }^{\text {a }}$ Data set identifier. C: Clay telescope secondary eclipse (magnitudes for each eclipse/night were separately normalized to zero median), H: Hankasalmi primary eclipse (normalized to zero median as above), O: MEarth out-of-eclipse (magnitudes in the instrumental system), P: MEarth primary eclipse (715 nm long-pass, normalized as above) and S: MEarth secondary eclipse (normalized as above).

${ }^{\mathrm{b}}$ Orbit number (from zero at $T_{0}$ ). Given only for the data sets containing eclipses. These are the numbers used in the figures showing the light curves.

${ }^{\mathrm{c}}$ Photometric zero-point correction applied by the differential photometry procedure. Note: this has already been included in the $I$ column and is for reference purposes only (e.g., detecting frames with large light losses due to clouds).

${ }^{\mathrm{d}}$ FWHM of the stellar images on the frame. Zero in cases where the FWHM could not be reliably estimated.

e Flag for detecting "meridian flip" on German equatorial mountings. Used only for the MEarth data, where the flag is 0 when the telescope was observing in the usual orientation for the east side and 1 for the west side of the meridian. Note that there is not a one-to-one relation with the sign of the hour angle because the MEarth mounts can track approximately $5^{\circ}$ across the meridian before needing to change sides of the pier, so this flag can sometimes be 0 even for (small) positive hour angle.

${ }^{\mathrm{f}}$ For the MEarth data only. Gives the "common mode" interpolated to the Julian date of the exposure. Derived from the average differential magnitude of all the M-dwarfs observed by all eight MEarth telescopes in a given time interval. This should be scaled and subtracted from the $I$ column to correct for the suspected variations in the MEarth bandpass with humidity and temperature (see the text).

(This table is available in its entirety in a machine-readable form in the online journal. A portion is shown here for guidance regarding its form and content.)

with a further eclipse (which was also a secondary) observed on UT 2010 June 8 with MEarth and the Clay telescope (see Section 2.2).

\subsection{Secondary Eclipses}

By the time the orbital ephemeris was well known, the observing season on LSPM J1112+7626 from Arizona was ending, and the weather had begun to deteriorate due to the arrival of the monsoon. We therefore took advantage of the high declination of our target and commenced an observing program to target the secondary eclipses using the Harvard University $0.4 \mathrm{~m}$ Landon T. Clay telescope, which is located on the roof of the Science Center on the Harvard campus near Harvard Square, Cambridge, Massachusetts. This instrument is normally used for undergraduate teaching. It is equipped with an e2v CCD47-10 $1024 \times 1024$ pixel back-illuminated CCD packaged by Apogee Instruments (model AP47), which was operated binned $2 \times 2$ for a final pixel scale of $\approx 1^{\prime \prime} .59$ pixel $^{-1}$ to reduce overheads.

The location of the telescope is extremely light polluted, and combined with the red colors of our target, we decided to use the $I$-band filter to mitigate the effects of sky background and atmospheric extinction. The filter available was built to the prescription of Bessell (1990b) using Schott RG9 glass to define the bandpass, and shall hereafter be referred to as $I_{\text {Bessell }}$. While this filter was intended to approximate the Cousins I passband (hereafter $I_{C}$ ), the combination of the glass filter and CCD quantum efficiency yields a system response with significant sensitivity at very red wavelengths not present in the original Cousins passband, and thus is a poor approximation to the Cousins filter for very red stars such as M-dwarfs. We shall return to this issue in Section 4.4.

Data were successfully gathered using the Clay telescope for the secondary eclipses on UT 2010 June 8, October 9, November 19, and December 30. The entire eclipse was visible for all but the June 8 event, although varying fractions of these were lost due to clouds. Exposure times were adjusted to keep the peak counts in the target below 30,000 to avoid saturation and ranged from 30 to $60 \mathrm{~s}$. The typical cadence was $50 \mathrm{~s}$ and the FWHM of the stellar images was 2-3 binned pixels. No guiding was available, so the target was re-centered manually approximately every 20 exposures to attempt to keep the drift below 5 pixels.

Data were reduced and light curves produced using standard procedures for CCD data, following the method outlined in Irwin et al. (2007). Two-dimensional bias frames were subtracted as there was some bias structure evident, and we used the overscan region to correct for any bias level variations. Dark frames of exposure matched to the target frames were subtracted to correct for the (significant) dark current and hot pixels at the $-20^{\circ} \mathrm{C}$ operating temperature of the $\mathrm{CCD}$, and the data were flat-fielded using twilight flat-fields in the usual way. No defringing or absolute calibration of the photometry were attempted. Fringing was clearly visible in the images, but at a low amplitude (3\% peak-to-peak) and should not have a significant effect on the photometry due to the stabilized telescope pointing.

We present the full light curve data set used in this paper in Table 1, and times of minimum light for the eclipses where a clear minimum was seen in Table 2.

\subsection{Primary Eclipse}

Using the timing of the single visit within the primary eclipse of UT 2010 April 3 from MEarth (see Section 2.1) combined with modeling of the secondary eclipses (Section 2.2) and radial velocities (Section 3) it was possible to predict the primary eclipse times with reasonable accuracy. During 2010-2011 full eclipses were observable at reasonable airmass $(<2.0)$ from eastern Europe, Scandinavia, and western Asia, with the high declination of the target favoring northerly latitudes during winter to maximize dark hours at reasonable airmass.

The primary eclipse was observed using the $40 \mathrm{~cm}$ telescope at Hankasalmi Observatory, Finland on UT 2011 January 15, starting approximately ten minutes before first contact, and continuing until well after last contact. Data were taken using 
Table 2

Times of Minimum Light

\begin{tabular}{lcccc}
\hline \hline $\begin{array}{l}\text { HJD_UTC } \\
\text { (days) }\end{array}$ & $\begin{array}{c}\text { Uncertainty } \\
(\mathrm{s})\end{array}$ & Cycle & Eclipse & Instrument \\
\hline 2455577.272644 & 7 & 7 & Primary & Hankasalmi \\
2455355.627938 & 41 & 1 & Secondary & Clay \\
2455478.725045 & 42 & 4 & Secondary & Clay \\
2455519.757412 & 31 & 5 & Secondary & Clay \\
2455560.789627 & 11 & 6 & Secondary & Clay \\
2455519.757351 & 16 & 5 & Secondary & MEarth \\
2455683.887000 & 20 & 9 & Secondary & MEarth \\
\hline
\end{tabular}

Notes. Estimated using the method of Kwee \& van Woerden (1956), over an interval of $\pm 10^{-3}$ in normalized orbital phase. These times of minimum are not used in our analysis (we fit for the ephemeris directly from the data) and are reported only for completeness.

a similar $I_{\text {Bessell }}$ filter to the secondary eclipse observations (see Section 2.2) with a front-illuminated Kodak KAF-1001E CCD packaged by Santa Barbara Instrument Group (model STL-1001E). This system response should be very similar to the one used for the secondary eclipses, but nonetheless, any bandpass mismatch is a potential concern for the accuracy of the photometric solution when combining data taken with different instruments, and will be discussed in more detail in Section 4.4.

Exposure times of $60 \mathrm{~s}$ were used, yielding a cadence of approximately two minutes, and the target was re-centered every ten exposures to keep the drift below $\approx 50$ pixels. Data were reduced using two-dimensional bias frames, and master dark and flat-field frames. Light curves were then derived using the same methods and software as for the other data sets.

\subsection{Out of Eclipse and Additional Secondary Eclipses}

Following the discovery of atmospheric water vapor induced systematic effects in the MEarth photometry (see Irwin et al. 2011), a customized $I$-band filter was procured for the start of the 2010-2011 season, with a bandpass designed to eliminate the strong telluric water vapor absorption at wavelengths $>900 \mathrm{~nm}$, by using an $895 \mathrm{~nm}$ (50\% transmittance point) interference cutoff on the same RG715 glass substrate as the original filter (where the long-pass glass filter defines the blue end of the bandpass). This was predicted to reduce the effect to largely insignificant levels, and the resulting system response approximates the Cousins $I$ bandpass (as tabulated by Bessell 1990b; see later in this section).

Observations of LSPM J1112+7626 using this new filter commenced on UT 2010 October 29 and continued until UT 2011 May 18. Exposures of $2 \times 97 \mathrm{~s}$ were used at 20 minute cadence for times out of eclipse. Five thousand two hundred and eighty-three useful data points were obtained at airmasses $<2$, after discarding 400 bad frames (pointing errors, severe light loss due to clouds, and large scatter in the zero-point solution for the differential photometry).

Partial secondary eclipses were obtained with the same MEarth telescope on UT 2010 November 19, UT 2011 February 9, and UT 2011 May 2. Due to known systematic effects caused by persistence in the MEarth CCDs, leading to offsets in magnitude depending on the cadence (see Berta et al. 2011), and to remove any residual from the out-of-eclipse variations, we allow these high-cadence observations to have different photometric zero points than the out-of-eclipse data (and each other) in the final light curve analysis.

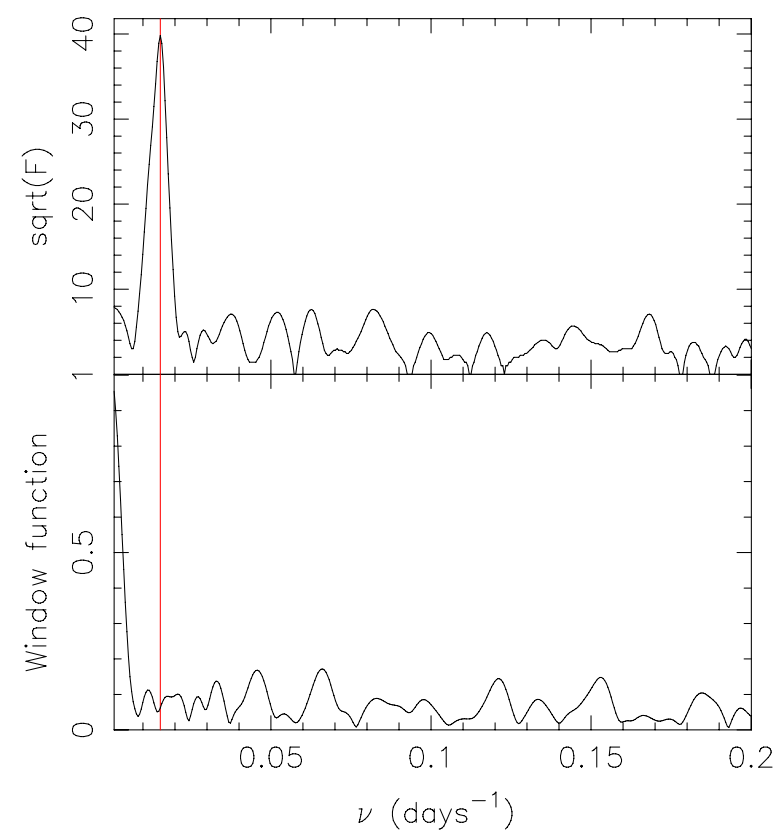

Figure 1. "F-test periodogram" for the MEarth 2010-2011 season out-ofeclipse observations, plotting the square root of the $F$-test statistic comparing the null hypothesis of no variation to the alternate hypothesis of a sinusoidal modulation, as a function of frequency (top) and the corresponding window function (bottom). The vertical line indicates the best-fitting period of 64.8 days. (A color version of this figure is available in the online journal.)

The out-of-eclipse data (excluding the high-cadence eclipse observations) were searched for periodic variations using the method outlined in Irwin et al. (2011). Correction for the "common-mode" effect was still performed, as we discovered during the season that replacing the filters had in fact increased the size (and changed the sign) of the systematic effect rather than eliminating it. This problem is still under investigation, but we suspect it is due to humidity and/or temperature dependence of the filter bandpass, an effect also seen in the original Sloan Digital Sky Survey (SDSS) Photometric Telescope filters (Doi et al. 2010).

After correcting for the "common-mode" effect, the MEarth data show a strong near-sinusoidal modulation with a 65 day period (see Figure 1; the data are shown in Section 4) and a peak-to-peak amplitude of approximately $0.02 \mathrm{mag}$. Marginal evidence for a similar periodicity is found in the 2009-2010 data, although the phase is not consistent and the amplitude appears to be much smaller. It is unclear if the amplitude change results from the differing filter systems, but the change in phase (if real) may be indicative of evolution of the photospheric spot patterns giving rise to the modulations.

An improvement resulting from the new MEarth filter is a greatly reduced color term when standardizing the photometry onto the Cousins system. The scatter in this relation also appears to be significantly lower, which may also result in part from improvements made to our flat-fielding strategy during the 2010-2011 season. The MEarth observing software automatically obtains measurements of equatorial photometric standard star fields from the catalog of Landolt (1992) every night, and these are used to derive photometric zero points and calibrated photometry. The typical scatter in these zero-point solutions on a photometric night is $0.02 \mathrm{mag}$. We find a typical atmospheric extinction of $0.05 \mathrm{mag} /$ airmass from MEarth data, which has been assumed henceforth. 
Table 3

Summary of the Photometric and Astrometric Properties of the LSPM J1112+7626 System

\begin{tabular}{lc}
\hline \hline Parameter & Value \\
\hline$\alpha_{2000}{ }^{\mathrm{a}, \mathrm{b}}$ & $11^{\mathrm{h}} 12^{\mathrm{m}} 42^{\mathrm{s}} .35$ \\
$\delta_{2000}{ }^{\mathrm{a}, \mathrm{b}}$ & $+76^{\circ} 26^{\prime} 56^{\prime \prime} \cdot 3$ \\
$\mu_{\alpha} \cos \delta^{\mathrm{b}}$ & $0^{\prime \prime} 131 \mathrm{yr}^{-1}$ \\
$\mu_{\delta}^{\mathrm{b}}$ & $-0^{\prime \prime} 108 \mathrm{yr}^{-1}$ \\
$g^{\mathrm{c}}$ & $15.618 \pm 0.010$ \\
$r^{\mathrm{c}}$ & $14.195 \pm 0.012$ \\
$z^{\mathrm{c}}$ & $12.027 \pm 0.012$ \\
$I_{\mathrm{C}}{ }^{\mathrm{d}}$ & $12.14 \pm 0.05$ \\
$J_{2 \mathrm{MASS}}{ }^{\mathrm{e}}$ & $10.591 \pm 0.023$ \\
$H_{2 \mathrm{MASS}}{ }^{\mathrm{e}}$ & $9.989 \pm 0.021$ \\
$K_{2 \mathrm{MASS}}{ }^{\mathrm{e}}$ & $9.728 \pm 0.017$ \\
\hline
\end{tabular}

Notes.

${ }^{a}$ Equinox J2000.0, epoch 2000.0.

b From Lépine \& Shara (2005).

c From SDSS Data Release 7. Note that these uncertainties do not account for variability and are therefore underestimates.

${ }^{\mathrm{d}}$ From Section 2.4.

e We quote the combined uncertainties from the 2MASS catalog, noting that the intrinsic variability of our target means in practice that these are underestimates.

A significant issue with the equatorial standard fields is they contain very few red stars suitable for defining the red end of the photometric response, and so color equations derived only from these equatorial fields may be significantly in error when attempting to standardize M-dwarf photometry. Thankfully, MEarth serendipitously targeted a number of M-dwarfs with photometry available on the Cousins system from Bessel (1990a). We selected all such objects in the 2010-2011 MEarth database with data available on photometric nights for calibration. Two objects with clearly unreliable MEarth data were discarded, leaving eight objects. The following color equation was derived from these stars, which span $I_{C}-K_{2 \text { MAss }}$ colors of 2.2-4.2:

$$
\begin{aligned}
I_{C}= & I_{\text {MEarth }}-(0.085 \pm 0.019) \\
& +(0.0204 \pm 0.0064)\left(I_{\text {MEarth }}-K_{2 \text { MASS }}\right) .
\end{aligned}
$$

In the case of LSPM J1112+7626, we estimate $I_{C}=12.14 \pm$ 0.05 using this relation, where we have inflated the uncertainty to account for variability and any errors introduced by the varying MEarth bandpass as discussed above. The observed $I_{C}-J, I_{C}-H$, and $I_{C}-K$ colors are later used to infer effective temperatures and luminosities for the members of the binary, and thus the distance to the system.

\subsection{Literature Data: Proper Motion and Photometry}

In Table 3, we summarize the photometric and astrometric system properties gathered from literature data and our photometry. This star lies within the area covered by the Sloan Extension for Galactic Understanding and Evolution stripe 1260 photometry, so we retrieved the point-spread function (PSF) magnitudes from Data Release 7 (Abazajian et al. 2009). This object is flagged as saturated in $i$-band (the image also shows a very unusual PSF and may be corrupted), and the $u$-band photometry of M-dwarfs in SDSS is corrupted by a well-documented red leak problem, so we omit these filters.

The moderately high proper motion of our target allows the contribution of any background objects to the system light to
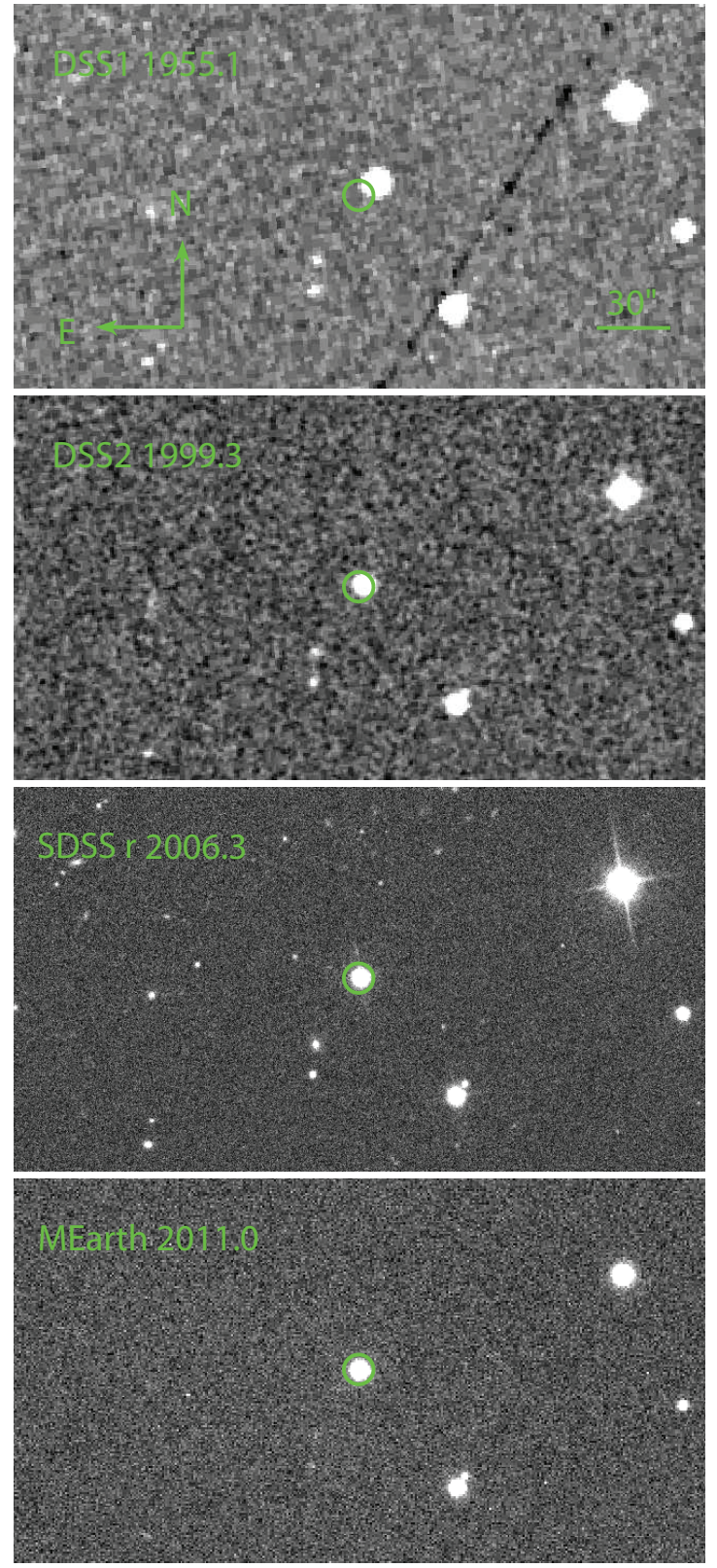

Figure 2. Images of LSPM J1112+7626 centered on the position as measured from the MEarth data. The circle shows the approximate position and size of the $8^{\prime \prime}$ (radius) photometric aperture used to derive the MEarth light curves. Data are from the first and second epoch Palomar sky surveys as provided by the Digitized Sky Survey (DSS; top and center panels), SDSS, and the MEarth master image (bottom panel). The approximate epochs are shown on the images, and all four panels have the same center, scale, and alignment on-sky.

(A color version of this figure is available in the online journal.)

be constrained using previous epochs of imaging. In Figure 2, we show imaging data with the position and size of the MEarth photometric aperture (which is representative of the aperture sizes used for all the photometry) overlaid. The DSS1 image indicates the aperture is unlikely to contain any significant flux from background objects, and the SDSS image (which has the highest angular resolution) does not appear elongated.

\subsection{Intermediate-resolution Optical Spectroscopy: Spectral Typing and Metallicity Constraints}

A single epoch intermediate-resolution $(R \simeq 1200)$ spectrum of our target was obtained using the FLWO $1.5 \mathrm{~m}$ Tillinghast 


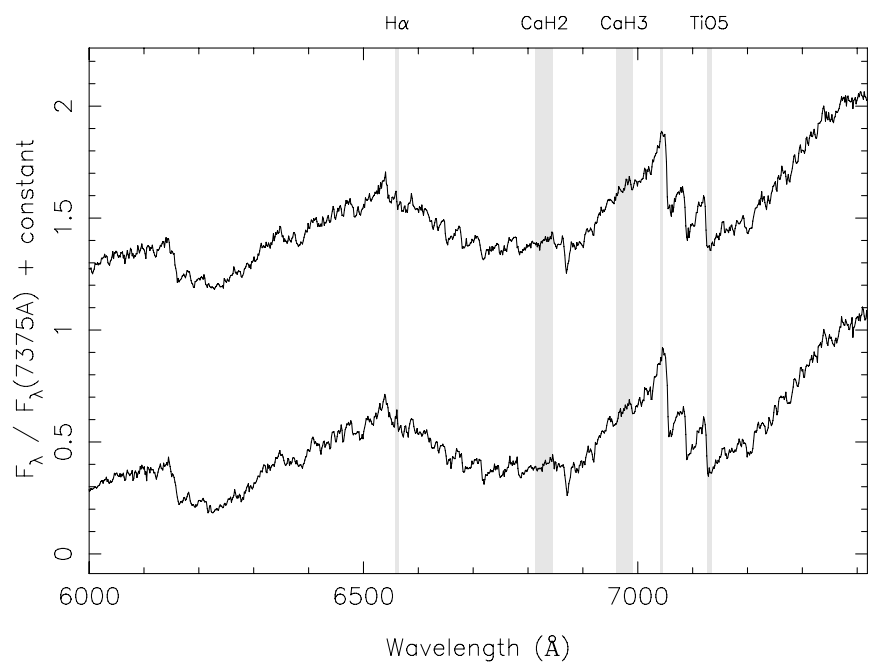

Figure 3. Intermediate-resolution FAST spectra of LSPM J1112+7626 showing the $\mathrm{TiO}$ and $\mathrm{CaH}$ molecular bands typically used for M-dwarf spectral classification. Two exposures were obtained and are shown offset in the vertical direction for clarity. Overlaid in gray are the locations of the $\mathrm{H} \alpha$ line, and the $\mathrm{CaH} 2$, $\mathrm{CaH} 3$, and TiO5 band indices defined by Reid et al. (1995).

reflector and FAST spectrograph (Fabricant et al. 1998) on UT 2011 March 16. The default setting of a $300 \mathrm{~g} \mathrm{~mm}^{-1}$ grating and $3^{\prime \prime}$ slit was used to obtain two exposures of $300 \mathrm{~s}$. The spectra were reduced using standard procedures for long slit spectra in IRAF $^{9}$ (Tody 1993), using an HeNeAr arc exposure taken in between the two target exposures to ensure accurate wavelength calibration. The two-dimensional spectra were divided by a flat field before extraction to remove the majority of the fringing seen at the reddest wavelengths. Relative flux calibration was performed using the spectrophotometric standard BD+26 2606. We show the resulting spectra in Figure 3.

Using the TiO5 band index defined by Reid et al. (1995), we find a composite spectral type of M3.7 (note that the uncertainty in this value is at least 0.5 subclass), and using THE HAMMER (Covey et al. 2007), we find M3-M4 (visual inspection indicates the observed spectrum is closer to M4). The spectral type and the measured colors are in good agreement using the empirical colors of Galactic disk M-dwarfs from Leggett (1992) for the Johnson/Cousins/CIT system and West et al. (2005) for the SDSS/Two Micron All Sky Survey (2MASS).

It is also clear from Figure 3 that no $\mathrm{H} \alpha$ line is seen in the spectrum. Such behavior is typical of inactive field dwarfs at these spectral types when observed at low resolution, where the equivalent width of the $\mathrm{H} \alpha$ absorption line becomes close to zero at M4-M5 (e.g., Gizis et al. 2002; see their Figure 5, noting that the TiO5 index was 0.42 for LSPM J1112+7626). The presence of $\mathrm{H} \alpha$ emission in M-dwarf spectra is usually indicative of high activity levels.

The relative strengths of the $\mathrm{TiO}$ and $\mathrm{CaH}$ bands in optical spectra have been used to identify and classify M-subdwarfs (Gizis 1997), and more generally proposed as a method for estimating metallicities of M-dwarfs (e.g., Woolf \& Wallerstein 2006). While detailed calibrations (e.g., using band indices) do not yet appear to be available, we performed a visual comparison between Figure 3 and the spectra shown in Gizis (1997). The strong $\mathrm{TiO}$ bands seen in our target indicate that it is not

\footnotetext{
9 IRAF is distributed by the National Optical Astronomy Observatory, which is operated by the Association of Universities for Research in Astronomy, Inc., under cooperative agreement with the National Science Foundation.
}

extremely metal poor. We therefore estimate it has metallicity $[\mathrm{Fe} / \mathrm{H}]>-1$ and is probably closer to solar (or greater).

\subsection{High-resolution Optical Spectroscopy: Radial Velocities}

High-resolution spectroscopic observations were obtained using the TRES fiber-fed échelle spectrograph on the FLWO $1.5 \mathrm{~m}$ Tillinghast reflector. We used the medium fiber $\left(2^{\prime \prime} .3\right.$ projected diameter), yielding a resolving power of $R \simeq 44,000$.

Observations commenced on UT 2010 May 1, and continued until UT 2011 April 13, with 43 epochs acquired. Total exposure times ranged from 45 to 80 minutes, broken into sequences of three or four individual exposures. The spectra were extracted using a custom built pipeline designed to provide precise radial velocities for échelle spectrographs. The procedures are described in more detail in Buchhave et al. (2010) and will be summarized briefly below.

In order to effectively remove cosmic rays, the sets of raw images were median combined. We used a flat field to trace the échelle orders and to correct the pixel-to-pixel variations in CCD response and fringing in the red-most orders, then extracted onedimensional spectra using the "optimal extraction" algorithm of Hewett et al. (1985) (see also Horne 1986). The scattered light in the two-dimensional raw image was determined and removed by masking out the signal in the échelle orders and fitting the inter-order light with a two-dimensional polynomial.

Thorium-Argon (ThAr) calibration images were obtained through the science fiber before and after each stellar observation. The two calibration images were combined to form the basis for the fiducial wavelength calibration. TRES is not a vacuum spectrograph and is only temperature controlled to $0.1^{\circ} \mathrm{C}$. Consequently, the radial velocity errors are dominated by shifts due to pressure, humidity, and temperature variations. In order to successfully remove these large variations $\left(>1.5 \mathrm{~km} \mathrm{~s}^{-1}\right)$, it is critical that the ThAr light travels along the same optical path as the stellar light and thus acts as an effective proxy to remove these variations. We have therefore chosen to sandwich the stellar exposure with two ThAr frames instead of using the simultaneous ThAr fiber, which may not exactly describe the induced shifts in the science fiber and can also lead to bleeding of ThAr light into the science spectrum from the strong argon lines, especially at redder wavelengths. The pairs of $\mathrm{ThAr}$ exposures were co-added to improve the signal-to-noise ratio, and centers of the ThAr lines found by fitting a Gaussian function to the line profiles. A two-dimensional fifth-order Legendre polynomial was used to describe the wavelength solution.

\section{SPECTROSCOPIC ANALYSIS}

Radial velocities were obtained using the two-dimensional cross-correlation algorithm TODCOR (Zucker \& Mazeh 1994), which uses templates matched to each component of a spectroscopic binary to simultaneously derive the velocities of both stars, and an estimate of their light ratio in the spectral bandpass.

We used a single epoch observation of Barnard's star (Barnard 1916; also known as Gl 699) taken on UT 2008 October 20 as the template for the TODCOR analysis. Barnard's star has a spectral type of M4 (Kirkpatrick et al. 1991), which should be a reasonable match to both components. Correlations were performed using a wavelength range of 7063-7201 $\AA$ in order 41 of the spectrum to derive the velocities, since this region contains strong molecular features (mostly from $\mathrm{TiO}$ ), which are rich in radial velocity information. We assume a barycentric radial velocity of $-110.3 \pm 0.5 \mathrm{~km} \mathrm{~s}^{-1}$ for Barnard's star (where the 
Table 4

Radial Velocity Data

\begin{tabular}{|c|c|c|c|c|}
\hline $\begin{array}{l}\text { HJD_UTC } \\
\text { (days) }\end{array}$ & Phase $^{b}$ & $\begin{array}{c}v_{1}{ }^{\mathrm{c}} \\
\left(\mathrm{km} \mathrm{s}^{-1}\right)\end{array}$ & $\begin{array}{c}v_{2}^{\mathrm{c}} \\
\left(\mathrm{km} \mathrm{s}^{-1}\right)\end{array}$ & $C_{\text {peak }}{ }^{\mathrm{d}}$ \\
\hline 2455317.6481 & 0.67269 & -49.45 & -78.49 & 0.560 \\
\hline 2455320.6671 & 0.74626 & -41.11 & -90.08 & 0.635 \\
\hline 2455321.6714 & 0.77074 & -38.58 & -93.77 & 0.643 \\
\hline 2455341.6568 & 1.25780 & -79.89 & -33.62 & 0.586 \\
\hline 2455342.6784 & 1.28270 & -79.29 & -34.71 & 0.506 \\
\hline 2455343.6545 & 1.30649 & -78.44 & -36.09 & 0.541 \\
\hline 2455344.6632 & 1.33107 & -78.21 & -36.76 & 0.577 \\
\hline 2455345.6848 & 1.35597 & -76.63 & -38.66 & 0.642 \\
\hline 2455346.6622 & 1.37979 & -75.23 & -40.34 & 0.536 \\
\hline 2455347.6488 & 1.40383 & -74.26 & -42.12 & 0.654 \\
\hline 2455348.6433 & 1.42807 & -72.38 & -44.19 & 0.602 \\
\hline 2455366.6561 & 1.86706 & -35.28 & -97.31 & 0.627 \\
\hline 2455367.6852 & 1.89214 & -37.23 & -96.02 & 0.588 \\
\hline 2455368.6748 & 1.91626 & -40.62 & -90.98 & 0.670 \\
\hline 2455369.6903 & 1.94101 & -44.54 & -84.52 & 0.576 \\
\hline 2455373.6462 & 2.03742 & -65.72 & -53.84 & 0.547 \\
\hline 2455374.6460 & 2.06178 & -69.12 & -48.82 & 0.662 \\
\hline 2455375.6455 & 2.08614 & -72.87 & -43.53 & 0.582 \\
\hline 2455581.9638 & 7.11433 & -75.90 & -39.72 & 0.750 \\
\hline 2455582.9270 & 7.13780 & -77.72 & -37.42 & 0.788 \\
\hline 2455583.8762 & 7.16093 & -79.01 & -35.40 & 0.811 \\
\hline 2455584.9160 & 7.18627 & -79.99 & -34.08 & 0.806 \\
\hline 2455586.9587 & 7.23606 & -80.51 & -33.75 & 0.854 \\
\hline 2455587.9329 & 7.25980 & -80.20 & -33.98 & 0.829 \\
\hline 2455605.9077 & 7.69786 & -46.45 & -82.16 & 0.816 \\
\hline 2455606.9374 & 7.72296 & -43.79 & -87.07 & 0.624 \\
\hline 2455607.9274 & 7.74709 & -41.08 & -90.00 & 0.797 \\
\hline 2455608.8926 & 7.77061 & -38.34 & -93.22 & 0.757 \\
\hline 2455611.8887 & 7.84363 & -34.67 & -99.11 & 0.731 \\
\hline 2455614.8719 & 7.91633 & -40.32 & -91.05 & 0.741 \\
\hline 2455615.8710 & 7.94068 & -44.85 & -84.66 & 0.822 \\
\hline 2455616.9096 & 7.96599 & -50.16 & -76.85 & 0.742 \\
\hline 2455617.8577 & 7.98910 & -55.18 & -69.76 & 0.788 \\
\hline 2455650.7940 & 8.79179 & -36.93 & -95.63 & 0.780 \\
\hline 2455651.7573 & 8.81526 & -35.56 & -97.88 & 0.732 \\
\hline 2455652.8008 & 8.84070 & -34.89 & -98.32 & 0.808 \\
\hline 2455656.7773 & 8.93761 & -44.06 & -85.15 & 0.814 \\
\hline 2455659.7417 & 9.00985 & -59.54 & -62.78 & 0.815 \\
\hline 2455664.7613 & 9.13218 & -77.22 & -38.03 & 0.826 \\
\hline
\end{tabular}

Notes.

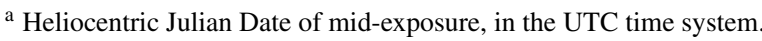

b Normalized orbital phase.

c Barycentric radial velocity of stars 1 and 2 .

d Peak normalized cross-correlation from TODCOR. These are later used for weighting the radial velocity points in the solution.

stated uncertainty reflects our estimate of the systematic errors), derived from presently unpublished CfA Digital Speedometer measurements spanning 17 years.

To derive the light ratio, we ran TODCOR using a range of fixed values of this quantity, seeking the maximum correlation over all epochs. In this way, consistency between the different epochs is enforced, in accordance with the expectation that the true value of this quantity should be (approximately) constant. This procedure improves the stability of the solution and minimizes systematic errors in the velocities. We find a best-fitting light ratio of $L_{2} / L_{1}=0.528 \pm 0.05$, where we adopt a conservative estimate of the uncertainty.

The radial velocities derived from our analysis are reported in Table 4. We omit four velocities with peak correlation values below $C_{\text {peak }}=0.5$ from the table and the remainder of the analysis as the low correlation values indicate that these velocities are likely to be unreliable or to exhibit large scatter. The uncertainty in the radial velocities (estimated from our fitting procedure; see Section 4) is $0.26 \mathrm{~km} \mathrm{~s}^{-1}$ for the primary, and $0.58 \mathrm{~km} \mathrm{~s}^{-1}$ for the secondary, with an additional systematic uncertainty, estimated to be $0.5 \mathrm{~km} \mathrm{~s}^{-1}$ (see above), in the velocity zero-point system.

We find no evidence for "peak pulling" effects close to the systemic velocity, which might indicate the need for additional rotational broadening of the template to match the target. Barnard's star is a very slow rotator (e.g., Benedict et al. 1998), so this and the high correlation values obtained without need for additional rotational broadening indicate both components of our target have low $v \sin i$, as expected.

\section{MODEL}

For EB systems showing moderate or high eccentricities, the commonly exploited property of being able to separate the photometric and spectroscopic solutions no longer holds as the eccentricity affects both. Typically, the orbital period $P$, epoch of primary eclipse $T_{0}$, and $e \cos \omega$ (where $e$ is eccentricity and $\omega$ is the argument of periastron) are better determined by the photometry (by eclipse timing; the latter is related to the departure of the secondary eclipses in phase from being exactly halfway between the primary eclipses, although note that the commonly used one-to-one relation between these quantities is approximate), and the $e \sin \omega$ component is usually best constrained by the velocities (but also affects the relative durations of the two eclipses, and in general eccentricity also influences their shape). Therefore, in order to leverage the best possible constraints on the system orbit, we adopt a joint solution of all the light curves and the radial velocities simultaneously. This also greatly simplifies the error analysis.

We proceed by first discussing the basic model and assumptions in Section 4.1, the method of solution in Section 4.2, and then the effects of four potentially important sources of systematic error in the system parameters we derive: spots (Section 4.3), bandpass mismatch (Section 4.4), limb darkening (Section 4.5), and third light (Section 4.6). In Section 4.7, we summarize our best estimates of the system parameters and their corresponding uncertainties.

\subsection{Basic Model and Assumptions}

To model the system, we used the light curve generator from the popular JKTEBOP code (Southworth et al. 2004a, 2004b), which is in turn based on the EB orbit program (EBOP; Popper \& Etzel 1981). This model approximates the stars using biaxial ellipsoids (Nelson \& Davis 1972), which is appropriate for well-detached systems such as the present one. We modified the model to perform the computations in double precision, to account for light travel time, which is necessary due to the large semimajor axis, and to incorporate a simple prescription for the effect of photospheric spots (discussed in Section 4.3). The light curve model was coupled with a standard Keplerian orbital model for the radial velocities (also including the light travel time). We correct the orbital period from the heliocentric frame to the system barycenter (Griffin et al. 1976) when computing the physical parameters, following the usual convention of reporting the orbital period $P$ as measured in the heliocentric frame (i.e., without the correction) in the tables.

We fit all of the Clay, Hankasalmi, and 2010-2011 season MEarth data, the radial velocities, and the spectroscopic light 
Table 5

Parameters and Priors Used in the Basic Model

\begin{tabular}{|c|c|c|c|}
\hline Parameter & Value & Prior $^{\mathrm{a}}$ & Description \\
\hline$J_{\text {MEarth }}$ & Varied & Uniform & Central surface brightness ratio (secondary/primary) in $I_{\text {MEarth }}$ \\
\hline$J_{\text {Bessell }}$ & Varied & Uniform & Central surface brightness ratio (secondary/primary) in $I_{\text {Bessell }}$ \\
\hline$\left(R_{1}+R_{2}\right) / a$ & Varied & Uniform & Total radius divided by semimajor axis \\
\hline$R_{2} / R_{1}$ & Varied & Uniform & Radius ratio \\
\hline $\cos i$ & Varied & Uniform (isotropic) & Cosine of orbital inclination \\
\hline$e \cos \omega$ & Varied & Uniform in $e, \omega$ & Eccentricity times cosine of argument of periastron \\
\hline$e \sin \omega$ & Varied & Uniform in $e, \omega$ & Eccentricity times sine of argument of periastron \\
\hline$L_{2} / L_{1}$ & $0.528 \pm 0.05$ & Gaussian & $I_{\text {MEarth-band light ratio }}$ \\
\hline$u_{1}$ & -0.0765 & Uniform & Primary limb darkening linear coefficient ${ }^{\mathrm{b}}$ \\
\hline$u_{1}^{\prime}$ & 1.0369 & Uniform & Primary limb darkening sqrt coefficient ${ }^{\mathrm{b}}$ \\
\hline$u_{2}$ & 0.0561 & Uniform & Secondary limb darkening linear coefficient ${ }^{b}$ \\
\hline$u_{2}^{\prime}$ & 0.9335 & Uniform & Secondary limb darkening sqrt coefficient ${ }^{\mathrm{b}}$ \\
\hline$\beta_{1}$ & 0.32 & $\ldots$ & Primary gravity darkening exponent ${ }^{c}$ \\
\hline$\beta_{2}$ & 0.32 & $\ldots$ & Secondary gravity darkening exponent ${ }^{\mathrm{c}}$ \\
\hline$\kappa_{1}$ & Computed & $\ldots$ & Primary reflection effect coefficient \\
\hline$\kappa_{2}$ & Computed & $\ldots$ & Secondary reflection effect coefficient \\
\hline$L_{3}$ & 0 & Uniform & Third light divided by total light ${ }^{\mathrm{b}}$ \\
\hline$\Phi$ & 0 & $\ldots$ & Tidal lead/lag angle \\
\hline$\delta$ & $2^{\circ}$ & $\ldots$ & Integration ring size ${ }^{\mathrm{d}}$ \\
\hline$F_{1}$ & Varied $^{\mathrm{e}}$ & Uniform & Primary rotation parameter \\
\hline$a_{1}$ & Varied $^{\mathrm{e}}$ & Uniform & Primary out-of-eclipse sine coefficient \\
\hline$b_{1}$ & Varied $^{\mathrm{e}}$ & Uniform & Primary out-of-eclipse cosine coefficient \\
\hline$F_{2}$ & Varied $^{\mathrm{e}}$ & Uniform & Secondary rotation parameter \\
\hline$a_{2}$ & Varied $^{\mathrm{e}}$ & Uniform & Secondary out-of-eclipse sine coefficient \\
\hline$b_{2}$ & Varied $^{\text {e }}$ & Uniform & Secondary out-of-eclipse cosine coefficient \\
\hline$q$ & Varied & Uniform & Mass ratio \\
\hline \multirow{2}{*}{$K_{1}+K_{2}$} & Varied & Modified Jeffreys & Sum of radial velocity semiamplitudes \\
\hline & & $K_{a}=0.02 \mathrm{~km} \mathrm{~s}^{-1}$ & Prior $P\left(K_{1}+K_{2}\right) \propto 1 /\left(K_{1}+K_{2}+K_{a}\right)$ \\
\hline$\gamma$ & Varied & Uniform & Systemic radial velocity \\
\hline$P$ & Varied & Uniform & Orbital period (heliocentric) \\
\hline$T_{0}$ & Varied & Uniform & Epoch of primary conjunction (HJD_UTC) \\
\hline$T_{\mathrm{sec}}$ & Computed & $\ldots$ & Epoch of secondary conjunction (HJD_UTC) \\
\hline$z_{l}$ & Varied & Uniform & Baseline magnitude for light curve $l^{\mathrm{f}}$ \\
\hline$s_{l}$ & Varied & Jeffreys & Error scaling factor for light curve $l^{\mathrm{f}}$ \\
\hline$k_{l}$ & Varied & Uniform & Airmass coefficient for light curve $l$ (where used $)^{\mathrm{f}}$ \\
\hline$C_{l}$ & Varied & Uniform & Common-mode coefficient for light curve $l$ (where used $)^{\mathrm{f}}$ \\
\hline$s_{r}$ & Varied & Jeffreys & Error (if $C_{\text {peak }}=1$ ) for radial velocity curve $r^{\mathrm{f}}$ \\
\hline
\end{tabular}

Notes.

${ }^{\text {a }}$ See Section 4.2 for discussion.

b Varied in later sections.

${ }^{\mathrm{c}}$ Values appropriate for convective atmospheres.

${ }^{\mathrm{d}}$ Our tests indicate this value results in a maximum error of $3 \times 10^{-5}$ mag in the eclipse depth (compared to a model with 0.1 ), which should be sufficient for our photometry.

${ }^{\mathrm{e}}$ Parameters varied only for one of the stars in each simulation.

${ }^{\mathrm{f}} l$ and $r$ are indices for the different light curves and radial velocities for each component of the system.

ratio simultaneously. We also include the single primary eclipse of UT 2010 April 3 to improve the constraint on the ephemeris, assuming the bandpass was approximately the same as the $I_{\text {Bessell }}$ filter (this makes little difference in practice due to the sparse coverage). The remainder of the MEarth 2009-2010 season data were omitted as these are of quite poor quality and were taken in a different bandpass from the remainder, so do not usefully constrain the model after accounting for the extra free parameters which must be added in order to fit them.

Table 5 summarizes the assumptions made in our light curve model. The light curve parameter set generally follows JKTEBOP and has been chosen to minimize correlations between parameters where possible. We add a term $k_{l}(X-1)$ to the model magnitudes, where $X$ is airmass, and $l$ is an index for the different light curves, to account for differential atmospheric extinction between the target and comparison stars, presumably due to color dependence. A different $k_{l}$ value was allowed for each light curve $l$. This has only been used for fitting the Clay secondary eclipse curves and 2010-2011 MEarth data (see Section 2.2), where it seems to be necessary to include this term to obtain an acceptable fit to the out-of-eclipse portions. We are unsure as to the exact origin of the baseline curvature in the Clay data, and note that the choice of a linear function of airmass is arbitrary and may not be correct. This is further evidenced by the inconsistent sign and magnitude of this term in Table 6 . We suggest the best approach to rectifying this problem would be to obtain higher quality secondary eclipse observations. 
Table 6

Derived Parameters and Uncertainties for the Four Spot Configurations with no Additional Spots

\begin{tabular}{|c|c|c|c|c|}
\hline \multirow[t]{2}{*}{ Parameter $^{\mathrm{a}}$} & \multicolumn{2}{|c|}{ Non-eclipsed Spots } & \multicolumn{2}{|c|}{ Eclipsed Spots } \\
\hline & Primary & Secondary & Primary & Secondary \\
\hline$J_{\text {MEarth }}$ & $0.7999 \pm 0.0064$ & $0.8004 \pm 0.0066$ & $0.7996 \pm 0.0064$ & $0.8423 \pm 0.0047$ \\
\hline$J_{\text {Bessell }}$ & $0.8255 \pm 0.0066$ & $0.8259 \pm 0.0069$ & $0.8252 \pm 0.0066$ & $0.8637 \pm 0.0043$ \\
\hline$\left(R_{1}+R_{2}\right) / a$ & $0.015548_{-0.000042}^{+0.000039}$ & $0.015551_{-0.000044}^{+0.000042}$ & $0.015550_{-0.000042}^{+0.000041}$ & $0.015661_{-0.000026}^{+0.000025}$ \\
\hline$R_{2} / R_{1}$ & $0.787 \pm 0.017$ & $0.788 \pm 0.018$ & $0.788 \pm 0.017$ & $0.888 \pm 0.020$ \\
\hline $\cos i$ & $0.004645_{-0.000112}^{+0.000103}$ & $0.004652_{-0.000115}^{+0.000109}$ & $0.004646_{-0.000111}^{+0.000105}$ & $0.005005_{-0.000058}^{+0.000051}$ \\
\hline$P$ (days) & $41.032363 \pm 0.000024$ & $41.032364 \pm 0.000024$ & $41.032365 \pm 0.000024$ & $41.032336 \pm 0.000025$ \\
\hline$T_{0}$ (HJD_UTC) & $2455290.04618 \pm 0.00018$ & $2455290.04618 \pm 0.00018$ & $2455290.04617 \pm 0.00018$ & $2455290.04637 \pm 0.00018$ \\
\hline$F_{1}$ & $0.6332 \pm 0.0019$ & $\ldots$ & $0.6331 \pm 0.0019$ & $\ldots$ \\
\hline$a_{1}(\mathrm{mag})$ & $0.00602 \pm 0.00067$ & $\ldots$ & $0.00599 \pm 0.00067$ & $\cdots$ \\
\hline$b_{1}$ (mag) & $0.00629 \pm 0.00062$ & $\ldots$ & $0.00632 \pm 0.00062$ & $\ldots$ \\
\hline$F_{2}$ & $\ldots$ & $0.6332 \pm 0.0019$ & $\ldots$ & $0.6327 \pm 0.0020$ \\
\hline$a_{2}(\mathrm{mag})$ & $\ldots$ & $0.0125 \pm 0.0014$ & $\ldots$ & $0.0098 \pm 0.0012$ \\
\hline$b_{2}(\mathrm{mag})$ & $\cdots$ & $0.0131 \pm 0.0013$ & $\ldots$ & $0.0109 \pm 0.0011$ \\
\hline$s_{\mathrm{OOE}}^{\mathrm{b}}$ & $1.469 \pm 0.014$ & $1.467 \pm 0.014$ & $1.467 \pm 0.014$ & $1.467 \pm 0.014$ \\
\hline$s_{\text {MEarth-20101119 }}$ & $1.191 \pm 0.058$ & $1.192 \pm 0.057$ & $1.191 \pm 0.058$ & $1.498 \pm 0.086$ \\
\hline$s_{\text {MEarth-20110209 }}$ & $1.548 \pm 0.087$ & $1.548 \pm 0.087$ & $1.549 \pm 0.087$ & $1.577 \pm 0.089$ \\
\hline$s_{\text {MEarth-20110502 }}$ & $1.194 \pm 0.055$ & $1.194 \pm 0.056$ & $1.193 \pm 0.055$ & $1.260 \pm 0.064$ \\
\hline$s_{\text {MEarth-Primary }}$ & $1.40 \pm 0.36$ & $1.39 \pm 0.35$ & $1.42 \pm 0.36$ & $1.39 \pm 0.35$ \\
\hline$s_{\text {Hankasalmi }}$ & $1.135 \pm 0.049$ & $1.136 \pm 0.049$ & $1.137 \pm 0.049$ & $1.143 \pm 0.050$ \\
\hline$s_{\text {Clay }-20100608}$ & $1.022 \pm 0.046$ & $1.023 \pm 0.046$ & $1.022 \pm 0.047$ & $1.034 \pm 0.046$ \\
\hline$s_{\text {Clay }-20101009}$ & $1.457 \pm 0.048$ & $1.455 \pm 0.048$ & $1.456 \pm 0.050$ & $1.498 \pm 0.050$ \\
\hline$s_{\text {Clay-20101119 }}$ & $1.177 \pm 0.038$ & $1.179 \pm 0.038$ & $1.178 \pm 0.038$ & $1.210 \pm 0.040$ \\
\hline$s_{\text {Clay-20101230 }}$ & $1.367 \pm 0.034$ & $1.367 \pm 0.034$ & $1.367 \pm 0.034$ & $1.357 \pm 0.033$ \\
\hline$k_{\text {MEarth }}$ & $-0.00659 \pm 0.00037$ & $-0.00658 \pm 0.00037$ & $-0.00659 \pm 0.00038$ & $-0.00686 \pm 0.00039$ \\
\hline$C_{\text {MEarth }}$ & $0.9834 \pm 0.0080$ & $0.9841 \pm 0.0080$ & $0.9842 \pm 0.0080$ & $0.9868 \pm 0.0080$ \\
\hline$k_{\text {Clay }-20100608}$ & $-0.0076 \pm 0.0031$ & $-0.0074 \pm 0.0031$ & $-0.0075 \pm 0.0031$ & $-0.0204 \pm 0.0032$ \\
\hline$k_{\text {Clay }-20101009}$ & $0.0136 \pm 0.0017$ & $0.0135 \pm 0.0017$ & $0.0135 \pm 0.0017$ & $0.0184 \pm 0.0017$ \\
\hline$k_{\text {Clay-20101119 }}$ & $0.0042 \pm 0.0014$ & $0.0041 \pm 0.0014$ & $0.0041 \pm 0.0014$ & $0.0031 \pm 0.0014$ \\
\hline$k_{\text {Clay-20101230 }}$ & $-0.0228 \pm 0.0014$ & $-0.0228 \pm 0.0014$ & $-0.0229 \pm 0.0014$ & $-0.0244 \pm 0.0014$ \\
\hline$e \cos \omega$ & $0.152408 \pm 0.000033$ & $0.152406 \pm 0.000034$ & $0.152409 \pm 0.000034$ & $0.152327 \pm 0.000035$ \\
\hline$e \sin \omega$ & $0.1823 \pm 0.0011$ & $0.1824 \pm 0.0012$ & $0.1823 \pm 0.0012$ & $0.1852 \pm 0.0012$ \\
\hline$q$ & $0.6958 \pm 0.0020$ & $0.6958 \pm 0.0020$ & $0.6958 \pm 0.0020$ & $0.6958 \pm 0.0022$ \\
\hline$\left(K_{1}+K_{2}\right)\left(\mathrm{km} \mathrm{s}^{-1}\right)$ & $55.602 \pm 0.087$ & $55.602 \pm 0.087$ & $55.603 \pm 0.087$ & $55.601 \pm 0.091$ \\
\hline$\gamma\left(\mathrm{km} \mathrm{s}^{-1}\right)^{\mathrm{c}}$ & $-61.071 \pm 0.027 \pm 0.5$ & $-61.071 \pm 0.027 \pm 0.5$ & $-61.070 \pm 0.027 \pm 0.5$ & $-61.068 \pm 0.028 \pm 0.5$ \\
\hline$s_{1}\left(\mathrm{~km} \mathrm{~s}^{-1}\right)$ & $0.125 \pm 0.015$ & $0.125 \pm 0.015$ & $0.125 \pm 0.015$ & $0.134 \pm 0.017$ \\
\hline$s_{2}\left(\mathrm{~km} \mathrm{~s}^{-1}\right)$ & $0.280 \pm 0.033$ & $0.279 \pm 0.032$ & $0.279 \pm 0.032$ & $0.290 \pm 0.034$ \\
\hline$i\left({ }^{\circ}\right)$ & $89.7339_{-0.0059}^{+0.0064}$ & $89.7335_{-0.0062}^{+0.0066}$ & $89.7338_{-0.0061}^{+0.0063}$ & $89.7132_{-0.0029}^{+0.0033}$ \\
\hline$e$ & $0.23763 \pm 0.00086$ & $0.23770 \pm 0.00088$ & $0.23762 \pm 0.00087$ & $0.23977 \pm 0.00090$ \\
\hline$\omega\left(^{\circ}\right)$ & $50.10 \pm 0.18$ & $50.12 \pm 0.19$ & $50.10 \pm 0.19$ & $50.56 \pm 0.19$ \\
\hline$a\left(R_{\odot}\right)$ & $43.825 \pm 0.069$ & $43.824 \pm 0.070$ & $43.825 \pm 0.070$ & $43.800 \pm 0.073$ \\
\hline$L_{2} / L_{1}$ & $0.485 \pm 0.025$ & $0.474 \pm 0.025$ & $0.486 \pm 0.025$ & $0.636 \pm 0.031$ \\
\hline$T_{\mathrm{sec}}\left(\mathrm{HJD} \_\right.$UTC) & $2455314.59517 \pm 0.00015$ & $2455314.59516 \pm 0.00015$ & $2455314.59516 \pm 0.00015$ & $2455314.59536 \pm 0.00015$ \\
\hline$M_{1}\left(M_{\odot}\right)$ & $0.3951 \pm 0.0022$ & $0.3950 \pm 0.0022$ & $0.3951 \pm 0.0022$ & $0.3944 \pm 0.0023$ \\
\hline$M_{2}\left(M_{\odot}\right)$ & $0.2749 \pm 0.0011$ & $0.2749 \pm 0.0011$ & $0.2749 \pm 0.0011$ & $0.2744 \pm 0.0012$ \\
\hline$\left(R_{1}+R_{2}\right)\left(R_{\odot}\right)$ & $0.6814_{-0.0020}^{+0.0020}$ & $0.6815_{-0.0021}^{+0.0021}$ & $0.6815_{-0.0021}^{+0.0020}$ & $0.6860_{-0.0016}^{+0.0016}$ \\
\hline$R_{1}\left(R_{\odot}\right)$ & $0.3815_{-0.0030}^{+0.0028}$ & $0.3814_{-0.0032}^{+0.0028}$ & $0.3814_{-0.0031}^{+0.0028}$ & $0.3635_{-0.0037}^{+0.0035}$ \\
\hline$R_{2}\left(R_{\odot}\right)$ & $0.2999_{-0.0043}^{+0.0044}$ & $0.3001_{-0.0044}^{+0.0047}$ & $0.3001_{-0.0043}^{+0.0045}$ & $0.3225_{-0.0042}^{+0.0043}$ \\
\hline
\end{tabular}

Notes.

${ }^{a}$ Parameter names as defined in Table 5 and in the text.

b The acronym OOE is used as shorthand for "out of eclipse."

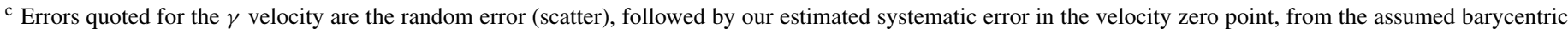
velocity of Barnard's star.

Gravity darkening coefficients were fixed at values appropriate for convective atmospheres (Lucy 1967), although gravity darkening is unimportant with the precision of the present light curves at such low rotation rates. The reflection effect was computed rather than fitting for it, although again the large semimajor axis means it is unimportant. 
We used a square root limb darkening law (Diaz-Cordoves \& Gimenez 1992) of the form

$$
\frac{I_{\lambda}(\mu)}{I_{\lambda}(1)}=1-u(1-\mu)-u^{\prime}(1-\sqrt{\mu})
$$

where $I_{\lambda}(\mu)$ is the specific intensity, and $\mu=\cos \theta$, where $\theta$ is the angle between the surface normal and the line of sight. As discussed by van Hamme (1993), the square root law is a better approximation to the specific intensity distribution given by model atmospheres of late-type stars in the NIR than the other common two-parameter laws (quadratic and logarithmic) implemented in ЈКтевоР. We also verified this by comparing the Claret (2000) four-parameter law (which we assumed to be the best representation of the original PHOENIX model) with the two-parameter laws for typical M-dwarf stellar parameters.

For our baseline model, we fixed the limb darkening coefficients to values for the $I_{C}$ filter derived from PHOENIX model atmospheres by Claret (2000), using $T_{\text {eff }}=3130 \mathrm{~K}$ for the primary, and $T_{\text {eff }}=3010 \mathrm{~K}$ for the secondary, $\log g=5.0$ and solar metallicity. We assume the same limb darkening law applies to all the $I$ filters (the error introduced by this assumption is examined in Section 4.5).

No evidence was found for any background sources in the photometric aperture (see Section 2.5), and the radial velocity observations do not show additional lines or velocity drifts that might indicate a comoving, physically associated companion with the EB system. We therefore assume no third light $\left(L_{3}=\right.$ 0 ), but see also Section 4.6.

\subsection{Method of Solution}

To derive parameters and error estimates, we adopt a variant of the popular Markov chain Monte Carlo analysis frequently applied in cosmology and for analysis of exoplanet radial velocity and light curve data (e.g., Tegmark et al. 2004; Ford 2005). We use adaptive Metropolis (Haario et al. 2001) rather than the standard Metropolis-Hastings method with the Gibbs sampler. The adaptive method allows adjustment of the proposal distributions while the chain runs and is thus simpler and faster to operate, obviating the need for manual tuning of the proposal distributions typical of the more conventional methods. This method uses a multivariate Gaussian proposal distribution to perturb all parameters simultaneously.

Compared to the simpler Monte Carlo and bootstrapping methods (e.g., as implemented in JKTEвор), these methods have an important advantage for the present case where a heterogeneous set of light curves and radial velocities must be analyzed: it is possible to estimate the appropriate inflation of the observational uncertainties self-consistently and simultaneously with the other model parameters (e.g., Gregory 2005). This allows the correct relative weighting of the various observational data sets to be decided essentially by their residuals from the best-fitting model, and the uncertainty in this weighting to be propagated to the final parameters. Of course, this assumes the correct model has been chosen for the data, so these methods must be used carefully.

In the Monte Carlo simulations, we used the standard Metropolis-Hastings acceptance criterion, accepting the new point with probability:

$$
P_{\text {acc }}=\left\{\begin{array}{l}
1, P\left(M_{i+1} \mid D\right) \geqslant P\left(M_{i} \mid D\right) \\
\frac{P\left(M_{i+1} \mid D\right)}{P\left(M_{i} \mid D\right)}, P\left(M_{i+1} \mid D\right)<P\left(M_{i} \mid D\right),
\end{array}\right.
$$

where $P\left(M_{i} \mid D\right)$ and $P\left(M_{i+1} \mid D\right)$ are the posterior probabilities of the previous and current points, respectively, $M_{i}$ represents the $i$ th model, and $D$ the data. The previous point in the chain was repeated if the new point was not accepted (this is required to correctly implement the method). The appropriate ratio of posterior probabilities ("Bayes factor") was

$$
\frac{P\left(M_{i+1} \mid D\right)}{P\left(M_{i} \mid D\right)}=\frac{P\left(M_{i+1}\right)}{P\left(M_{i}\right)} \exp \left(-\Delta \chi^{2} / 2\right)
$$

where the factors in the right-hand side are the ratio of prior probabilities and the usual (least squares) likelihood ratio, respectively.

Priors assumed for each parameter are detailed in Table 5 and are chosen to be uninformative. In most cases, this choice is a uniform improper prior (labeled "uniform" in the table). For the orbital inclination, we adopt a uniform prior on $\cos i$, which produces an isotropic distribution of orbit normals, and for the eccentricity, we use a uniform prior on $e$ and $\omega$, rather than on the jump parameters $e \cos \omega$ and $e \sin \omega$ (the latter would produce a prior in $e, \omega$ proportional to $e$; e.g., Ford 2006).

As discussed by Gregory (2005), the appropriate choice of uninformative prior for "scale parameters" such as radial velocity amplitude or orbital period is a Jeffreys prior (or a modified Jeffreys prior for parameters which can be zero). This enforces scale invariance (equal probability per decade). In the present case, some of these parameters (particularly the period) are so well-determined the choice of prior is unimportant, so for simplicity we have only used non-uniform priors on the less well-determined parameters where the prior is important.

As discussed earlier, and in Gregory (2005), it is possible to fit for the appropriate inflation of the observational uncertainties simultaneously with the model parameters. This has been done via the $s_{k}$ parameters shown in Table 5. It is conventional to apply this inflation by multiplication for light curves, and for radial velocity (RV) by adding an extra "jitter" contribution in quadrature. We follow these conventions here. Note that additional factors appear in the likelihood ratio of Equation (2) when doing this, which have been subsumed into the $\Delta \chi^{2}$ in the equation.

For the RV, it is difficult to estimate reliable uncertainties from cross-correlation functions, so instead we derive them during the simulation. We weight each point by $C_{\text {peak }}^{2}$ (the square of the peak normalized cross-correlation value; see Table 4), where we find $C_{\text {peak }}$ is approximately proportional to the signal-to-noise ratio in typical observations at similar signal to noise and peak correlation values to those seen in this work. This procedure is essentially equivalent to photon weighting, except the crosscorrelation accounts for all sources of uncertainty rather than only photon noise. The uncertainty corresponding to a peak correlation of unity is derived during the simulation, and we allow separate values of this quantity for each star, labeled $s_{1}$ for the primary, and $s_{2}$ for the secondary, in the tables. The resulting per-data-point uncertainties used in the fitting are given by $\sigma_{i j}=s_{i} / C_{\text {peak }, j}$ for the $i$ th star and $j$ th radial velocity point.

All Monte Carlo simulation results reproduced in this paper are derived from chains of $2 \times 10^{6}$ points in length, where the first $10 \%$ of the points were used to initialize the parameter covariance matrix for the adaptive Metropolis method (starting from the initial parameters and covariance matrix derived using a Levenberg-Marquardt minimization ${ }^{10}$ ), and the next $40 \%$ were used to "burn in" the chain. This was found to be sufficient to

\footnotetext{
$\overline{10}$ http://www.ics.forth.gr/ lourakis/levmar/
} 
ensure the chains were very well converged, and all of these first $50 \%$ of the points were discarded, leaving the remainder $\left(10^{6}\right.$ points) for parameter estimation. Correlation lengths in all parameters were $<200$ points.

We report the median and $68.3 \%$ central confidence intervals as the central value and uncertainty for all parameters. Reduced $\chi^{2}$ values for all the model fits were unity by construction.

\subsection{Spots}

In LSPM J1112+7626, the presence of spots is clearly indicated by the observed out-of-eclipse modulation described in Section 2.4.

Spots present severe difficulty for the analysis of EB light curves because they cause systematic errors in the measurements derived from the eclipses, principally their depths, which determine the geometric parameters $J,\left(R_{1}+R_{2}\right) / a$, and $i$ (and by extension, $\left.R_{2} / R_{1}\right)$. Spots occulted during eclipse temporarily reduce the depth as they are crossed, and spots not occulted during eclipse increase it, because the surface brightness of the photosphere under the eclipse chord is greater than would be inferred from the out-of-eclipse baseline level.

The difficulty in solving for physical system parameters arises because the true spot distribution is not usually known. Out-of-eclipse modulations, and in non-synchronized systems, modulation of the eclipses themselves, are sensitive only to the longitudinal inhomogeneity in the spot distribution, except in special cases where spots are crossed during eclipse and cause detectable deviations from the usual light curve morphology (this can be difficult to detect in systems with radius ratios close to unity, because the deviations then last a large fraction of the eclipse duration). Any longitudinally homogeneous component, such as a polar spot viewed equator-on, or a homogeneous surface coverage of small spots, cannot usually be detected from light curves.

This can cause undetected systematic errors in the radii and effective temperatures derived from the light curves. Morales et al. (2010) discuss this issue in some detail for the bestmeasured literature EBs, finding this effect could produce up to $6 \%$ systematic errors in the radii for stars with spots of $30 \%$ filling factor, when the spots are concentrated at the pole.

The influence of the spectroscopic light ratio is not commonly discussed with regard to spots, but this is important because it provides complementary information to the photometry, on the difference in spot coverage between the two stars. For example, consider the case of a large coverage of longitudinally homogeneous, polar spots that are not crossed during eclipse. If these spots are distributed on both stars, the light ratio is unaffected but both eclipses will appear deeper. However, if the spots are on only one star, the effect on the photometry is the same, but the light ratio is altered because the spotted star appears darker. This will produce a discrepancy between the light ratio derived only from the light curve parameters, and the spectroscopic value. More generally, it is possible to place constraints on the difference in the overall spot coverage between the binary components, using the spectroscopic information.

One unusual feature of the present system among low-mass EBs showing signs of spots is the spin and orbit appear to not be synchronized. Because of this, it is possible to measure eclipses at different rotational phases, and thus different spottedness of the visible stellar hemispheres. While this does not necessarily resolve the difficulty of determining the longitudinally homogeneous component of the spot distribution, it does provide additional information on the longitudinally in- homogeneous component, specifically which star the spots are located on. This information is not usually available if only outof-eclipse modulations are seen. It is common to assume the spots are on both binary components, which may be reasonable for near-equal mass systems at short periods where tidal synchronization is expected to have occurred, but this is not a reasonable assumption for LSPM J1112+7626, where only one out-of-eclipse modulation is seen. The observed rotational evolution of M-dwarfs (e.g., Irwin et al. 2011) indicates that it is extremely unlikely the two binary components could have the same rotation period (and phase) by chance in the absence of tidal effects, which are not expected.

Unfortunately, at the time of writing, only a single primary eclipse is available, so we are unable to take full advantage of the non-synchronized spin and orbit at present. Also, while multiple secondary eclipses were observed, and residuals from our best-fitting model (assuming no changes in eclipse shape or depth) are seen, it is not clear if many of these are simply the result of systematic errors in the photometry, given that equally large deviations are seen out of eclipse. Constraining the spot properties by this method will be an important area for future work, and observing in multiple bandpasses would be advantageous as it may provide information on the spot temperatures.

\subsubsection{Spot Model}

Conventionally, a Roche model such as the one implemented in the popular Wilson-Devinney program (Wilson \& Devinney 1971) would be used to model a system with spots as these usually include a circular spot model and perform the necessary surface integrals over the stars; however, the treatment of proximity effects is completely unnecessary in the present system, and these models are extremely computationally intensive, especially for systems with eccentric orbits, which would make a detailed Monte Carlo based error analysis prohibitive.

It is also not clear that the circular spot model with a small number of spots is realistic for late-type dwarfs. Observed light curves very rarely show the characteristic "eclipse-like" features with flat baseline at maximum light, as would be predicted for a small number of near-equatorial spots. This indicates either that these objects have very large, polar spots (e.g., Rodono et al. 1986), such that some of the spot is always in view to the observer to produce the continuous photometric modulations seen in light curves, or that the surfaces of these objects have many small spots (e.g., Barnes \& Collier Cameron 2001; Barnes et al. 2004) with the photometric modulations arising from a longitudinal inhomogeneity of the spot distribution. Modeling an ensemble of spots, as in the latter case, in detail would be prohibitive, as even single spot models are usually degenerate given limited light curve data. In our case, the degeneracies in fitting spot models would be further exacerbated by the availability of only single-band light curve information, meaning spot temperature and size would be essentially degenerate.

Given these difficulties, we take an alternative approach for the solution presented in this paper. Rather than trying to model the unknown spot distribution in detail, we simply attempt to incorporate the effect of spots into the uncertainties on the final parameters. We consider two models: (1) spots on the non-eclipsed part of the photosphere on both stars and (2), a case intended to approximate the effect of spots covering the whole photosphere, again for both stars. In both cases, the models are required to reproduce the observed out-of-eclipse modulation. It is necessary 

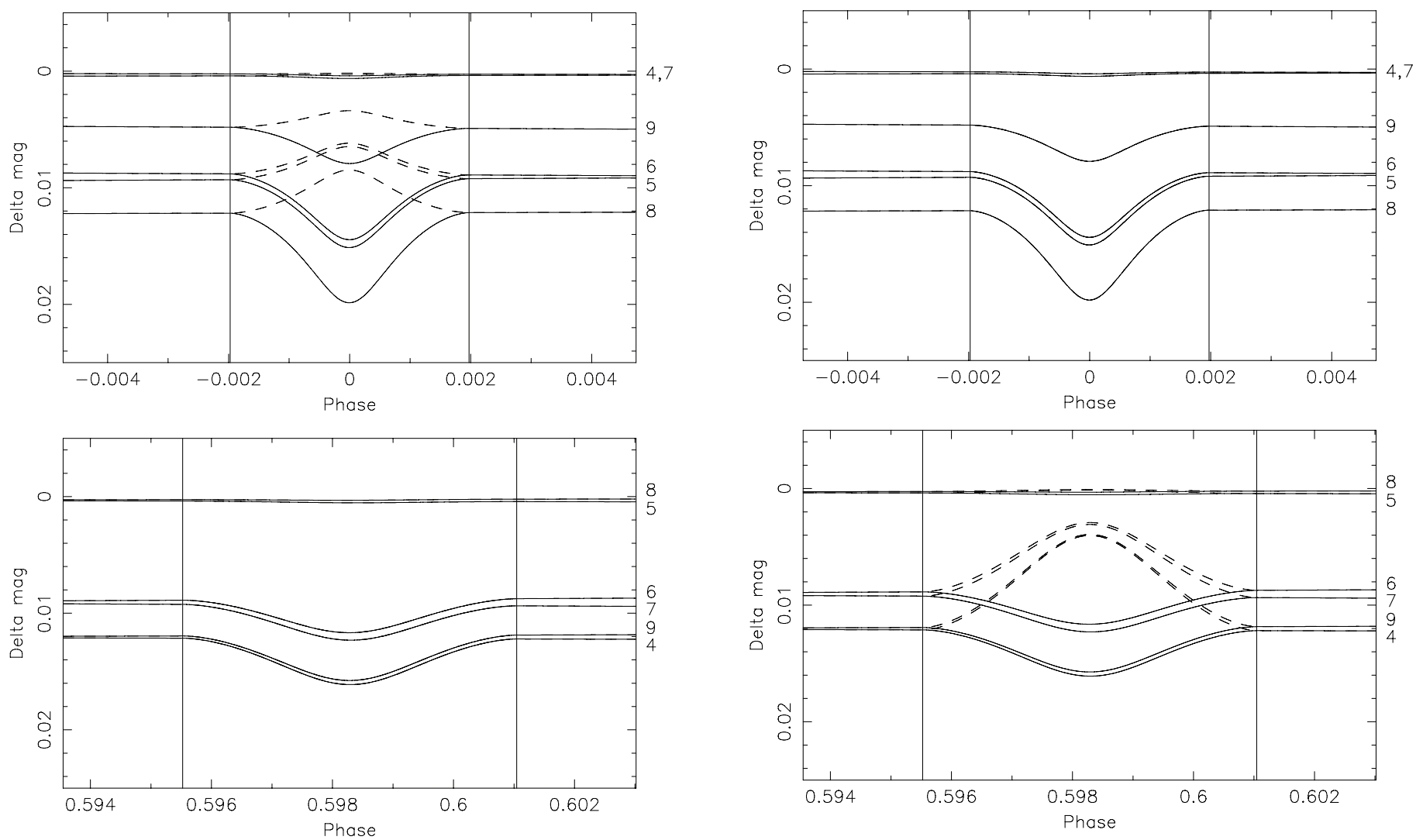

Figure 4. Predicted deviation in the primary (top) and secondary (bottom) eclipses resulting from spots on the primary star. The solid curves correspond to hypothesis (1) where the spots are not eclipsed, and the dashed curves to (2) where they are eclipsed. The two sets of curves are identical in the lower panel. The cycle numbers (integer part of the normalized orbital phase) are shown on the right of each panel.

to run two models in each spotted case because it is not known which star the out-of-eclipse modulation originates from.

We assume a simple sinusoidal form for the modulations, which appears to be an adequate description of the available light curve data. The functional form adopted was

$$
\frac{\Delta L_{i}}{L_{i}}=a_{i} \sin \left(\frac{2 \pi F_{i} t}{P}\right)+b_{i} \cos \left(\frac{2 \pi F_{i} t}{P}\right)-\sqrt{a_{i}^{2}+b_{i}^{2}},
$$

where $t$ is time, $L_{i}$ is the light from star $i, a_{i}$ and $b_{i}$ are constants expressing the amplitude and phase of the modulation, and $F_{i}$ is the "rotation parameter," the ratio of the rotation frequency to the orbital frequency. This expression is constructed to yield $\Delta L_{i}=0$ at maximum light, corresponding to the conventional assumption that no spots are visible when the star is brightest, which gives the minimum spot coverage necessary to reproduce the observed modulations.

JKTEBOP computes the final system light (and thus the change in magnitude) by summing the out-of-eclipse light and then subtracting the eclipsed light. By modulating only the out-ofeclipse light in this summation using Equation (3), hypothesis (1) can be implemented and hypothesis (2) can be implemented by also modulating the eclipsed light (changing the surface brightness under the eclipse chord) when the eclipsed star is star $i$.

\subsubsection{Results}

The effect of applying our spot model on the eclipses is shown in Figures 4 and 5, which were calculated using the parameters

Figure 5. Predicted deviation in the primary (top) and secondary (bottom) eclipses resulting from spots on the secondary star. Curves as Figure 4 . The two sets of curves are identical in the upper panel.

of LSPM J1112+7626, comparing a model with spots to a model with identical physical parameters, but without spots. This figure demonstrates that observing multiple eclipses at high precision would allow the star hosting the spots to be identified.

We fit all four spotted models to the full data set for LSPM $\mathrm{J} 1112+7626$ using the procedures already described. The results are given in Table 6 , and Figures 6-8 show the data with a representative model, corresponding to hypothesis (1), the "noneclipsed spots" model, on the primary, overplotted.

The 2010 November 19 partial secondary eclipse from MEarth (numbered 5 in the figure) provides the most discriminating power between the various models, particularly taken in conjunction with the 2011 May 2 event (numbered 9), which dominates the fit. As shown in Figure 7, these were taken at opposite extremes of the out-of-eclipse variation, with the 2010 November 19 event at maximum light and the 2011 May 2 event close to minimum light. Examining the sizes of the $s$ parameter in Table 6, the "eclipsed spots" model on the secondary, which is the only model presenting a significant detectable deviation in the secondary eclipses (as shown in Figure 5) is disfavored by the data (see Figure 9), having an $s_{\text {MEarth-20101119 value larger by }}$ almost $3 \sigma$ than the "non-eclipsed spots" model on the secondary. Indeed, the observations indicate that this eclipse needs to be made as shallow as possible in the model in order to produce a good fit, possibly even slightly shallower than the "non-eclipsed spots" model is able to produce (the discrepancy may arise from the imperfect modeling of the out-of-eclipse variation by a sinusoid). The depth of this eclipse therefore argues that if the spots causing the out-of-eclipse modulation are on the secondary, they should be at latitudes not crossed by the eclipse chord. 

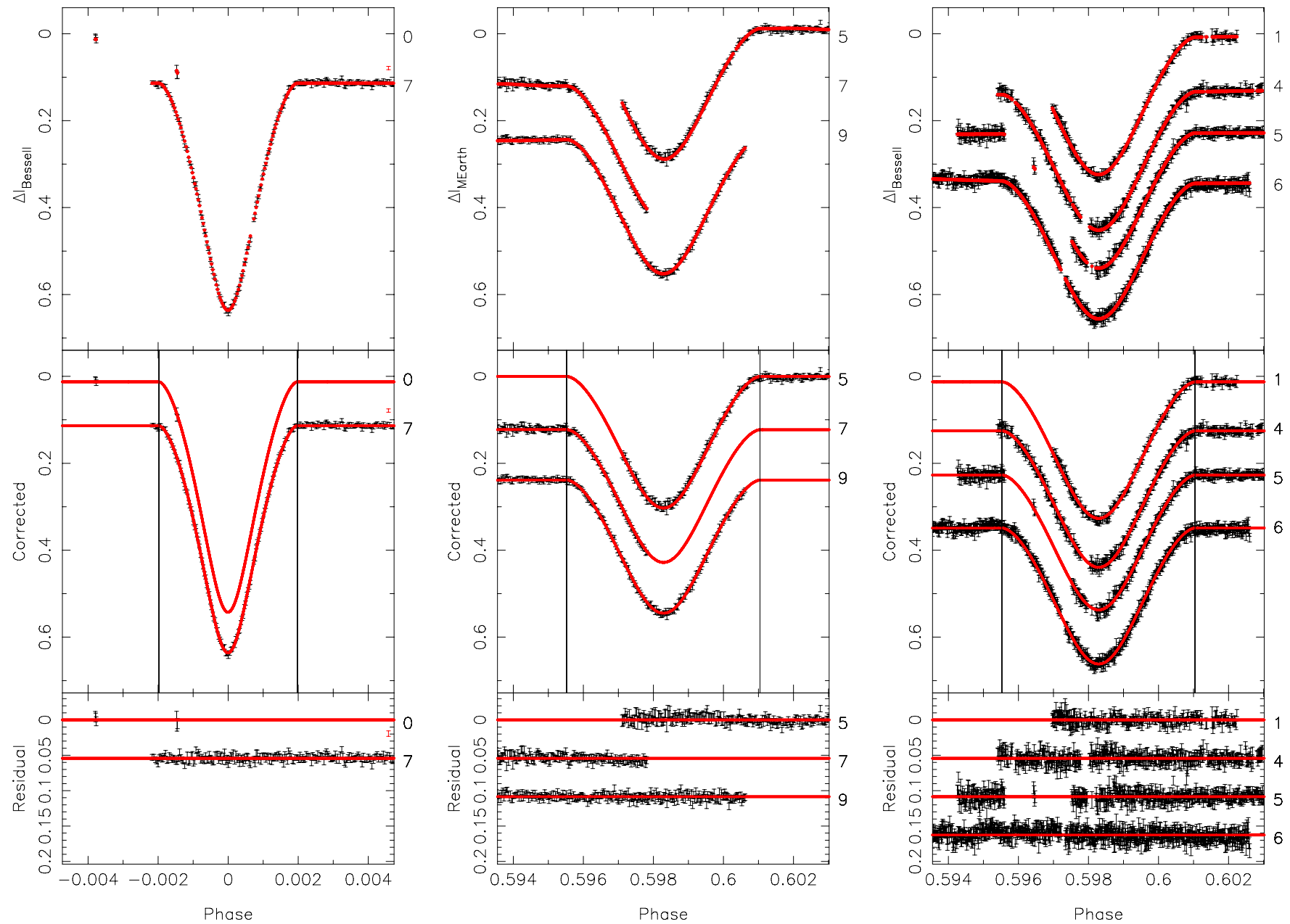

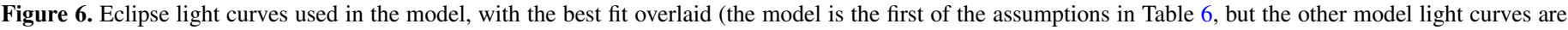

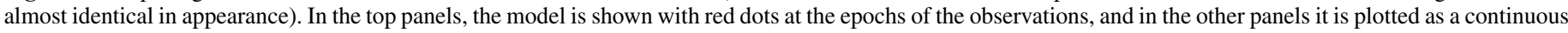

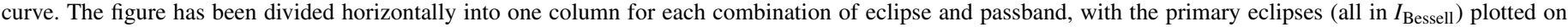

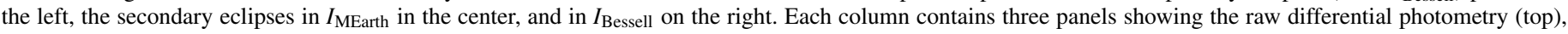

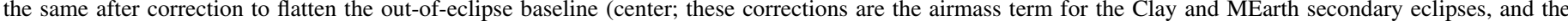

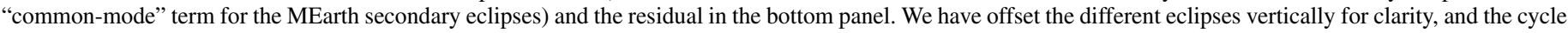
number (integer part of the normalized orbital phase) is shown on the right. The vertical bars indicate the approximate locations of the first and last contact points.

(A color version of this figure is available in the online journal.)

It is interesting to note that, because the radius ratio is quite close to unity, the range of allowed combinations of spot latitude and inclination angle for the secondary that would explain the observed out-of-eclipse modulations is therefore quite narrow, compared to the much wider range of possibilities on the primary that are still allowed. This mildly argues in favor of spots on the primary as being the more likely explanation, purely from geometry.

Nevertheless, with the presently available data, the other models do not show any clear disagreement with the observations, so we consider (from a purely observational point of view) that all three are equally likely at present.

\subsubsection{Effect of Additional Spots}

The model we have considered so far uses the minimum spot coverage necessary to reproduce the observed out-of-eclipse modulations. As noted, e.g., by Barnes et al. (2011) and other authors, studies of cool stars sensitive to the total spot coverage typically find much higher filling factors than photometric spot models. Therefore, it is likely these stars have a significant longitudinally homogeneous spot component.
In this section, we consider the effect of adding additional spots in a longitudinally homogeneous fashion where they would not be detectable through modulations in the photometry. For simplicity, we have placed these spots on the same star and at the same latitudes as the longitudinally inhomogeneous component, noting that we are predominantly interested in the effect on the component radii, where it does not matter which of the possible locations is used for the longitudinally inhomogeneous spot component.

As discussed earlier, the spectroscopic light ratio is sensitive to differences in the overall spot coverage between the two stars in some cases. We have explored this limit, and the effect of adding varying quantities of spots on the component radii, by subtracting an extra term $c_{i}$ in Equation (3) representing the fraction of stellar light removed by the longitudinally homogeneous spot component.

For single late-type active dwarfs, it is typical to find spot filling factors of $20 \%-50 \%$ from observations sensitive to the entire surface spot coverage (e.g., O'Neal et al. 2004), although we note the available information for M-dwarfs is very limited. Photometric observations of M-dwarfs indicate spot temperature 

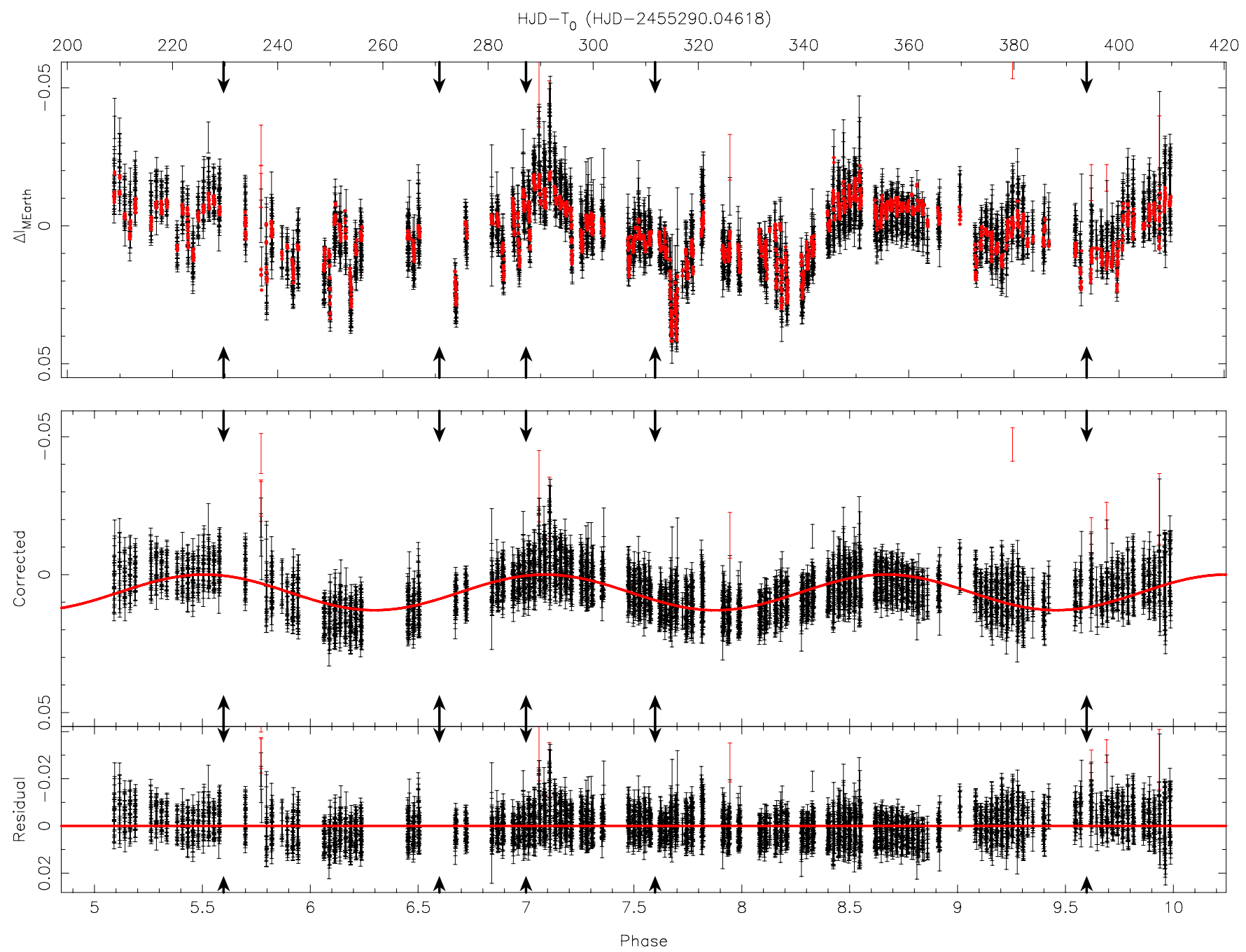

Figure 7. MEarth 2010-2011 season out-of-eclipse light curve. Vertical panels as Figure 6, with the best-fitting sinusoidal model overplotted. Arrows indicate the phases corresponding to the observed eclipses.

(A color version of this figure is available in the online journal.)

Table 7

Derived Parameters and Uncertainties Showing the Effect of Additional Spots on the Primary

\begin{tabular}{|c|c|c|c|c|c|c|}
\hline \multirow[t]{2}{*}{ Parameter } & \multicolumn{3}{|c|}{ Non-eclipsed Spots } & \multicolumn{3}{|c|}{ Eclipsed Spots } \\
\hline & $c_{1}=0$ & $c_{1}=0.1$ & $c_{1}=0.2$ & $c_{1}=0$ & $c_{1}=0.1$ & $c_{1}=0.2$ \\
\hline$J_{\text {MEarth }}$ & $0.7999 \pm 0.0064$ & $0.8302 \pm 0.0066$ & $0.8724 \pm 0.0074$ & $0.7996 \pm 0.0064$ & $0.7196 \pm 0.0060$ & $0.6396 \pm 0.0051$ \\
\hline$J_{\text {Bessell }}$ & $0.8255 \pm 0.0066$ & $0.8583 \pm 0.0068$ & $0.9042 \pm 0.0079$ & $0.8252 \pm 0.0066$ & $0.7427 \pm 0.0062$ & $0.6602 \pm 0.0054$ \\
\hline$\left(R_{1}+R_{2}\right) / a$ & $0.015548_{-0.000042}^{+0.000039}$ & $0.015632_{-0.000039}^{+0.000039}$ & $0.015775_{-0.000039}^{+0.000037}$ & $0.015550_{-0.000042}^{+0.000041}$ & $0.015551_{-0.000044}^{+0.000042}$ & $0.015550_{-0.000042}^{+0.000041}$ \\
\hline$R_{2} / R_{1}$ & $0.787 \pm 0.017$ & $0.766 \pm 0.016$ & $0.756 \pm 0.017$ & $0.788 \pm 0.017$ & $0.788 \pm 0.018$ & $0.787 \pm 0.017$ \\
\hline $\cos i$ & $0.004645_{-0.000112}^{+0.000103}$ & $0.005219_{-0.000086}^{+0.000083}$ & $0.005861_{-0.000072}^{+0.000065}$ & $0.004646_{-0.000111}^{+0.000105}$ & $0.004646_{-0.000116}^{+0.000109}$ & $0.004644_{-0.000112}^{+0.000107}$ \\
\hline$i\left(^{\circ}\right)$ & $89.7339_{-0.0059}^{+0.0064}$ & $89.7010_{-0.0047}^{+0.0049}$ & $89.6642_{-0.0037}^{+0.0041}$ & $89.7338_{-0.0061}^{+0.0063}$ & $89.7338_{-0.0063}^{+0.0067}$ & $89.7339_{-0.0061}^{+0.0064}$ \\
\hline$L_{2} / L_{1}$ & $0.485 \pm 0.025$ & $0.531 \pm 0.025$ & $0.611 \pm 0.032$ & $0.486 \pm 0.025$ & $0.486 \pm 0.026$ & $0.486 \pm 0.025$ \\
\hline$\left(R_{1}+R_{2}\right)\left(R_{\odot}\right)$ & $0.6814_{-0.0020}^{+0.0020}$ & $0.6847_{-0.0020}^{+0.0020}$ & $0.6904_{-0.0021}^{+0.0020}$ & $0.6815_{-0.0021}^{+0.0020}$ & $0.6815_{-0.0021}^{+0.0021}$ & $0.6815_{-0.0021}^{+0.0020}$ \\
\hline$R_{1}\left(R_{\odot}\right)$ & $0.3815_{-0.0030}^{+0.0028}$ & $0.3878_{-0.0029}^{+0.0026}$ & $0.3934_{-0.0033}^{+0.0030}$ & $0.3814_{-0.0031}^{+0.0028}$ & $0.3814_{-0.0032}^{+0.0029}$ & $0.3814_{-0.0031}^{+0.0028}$ \\
\hline$R_{2}\left(R_{\odot}\right)$ & $0.2999_{-0.0043}^{+0.0044}$ & $0.2969_{-0.0039}^{+0.0042}$ & $0.2970_{-0.0043}^{+0.0045}$ & $0.3001_{-0.0043}^{+0.0045}$ & $0.3001_{-0.0044}^{+0.0047}$ & $0.3000_{-0.0043}^{+0.0045}$ \\
\hline
\end{tabular}

contrast of up to $10 \%$ (e.g., Rockenfeller et al. 2006, who find $4 \%-8 \%$ ). This yields a decrement of approximately $10 \%-20 \%$ in the $I$-band stellar light, assuming parameters appropriate to the present system, with the lower end of the range presumably more typical for less active stars. We therefore consider values of $c_{i}=0.1$ and 0.2 . The latter corresponds approximately to the spot levels discussed by Morales et al. (2010) for the existing short-period EBs. The results are given in Tables 7 and 8.

First, it is clear from Table 7 that adding additional "eclipsed spots" has no effect on the radii or light ratio and merely alters the surface brightness ratio (this means it also changes the effective temperature ratio). This is also true when placing these spots on 


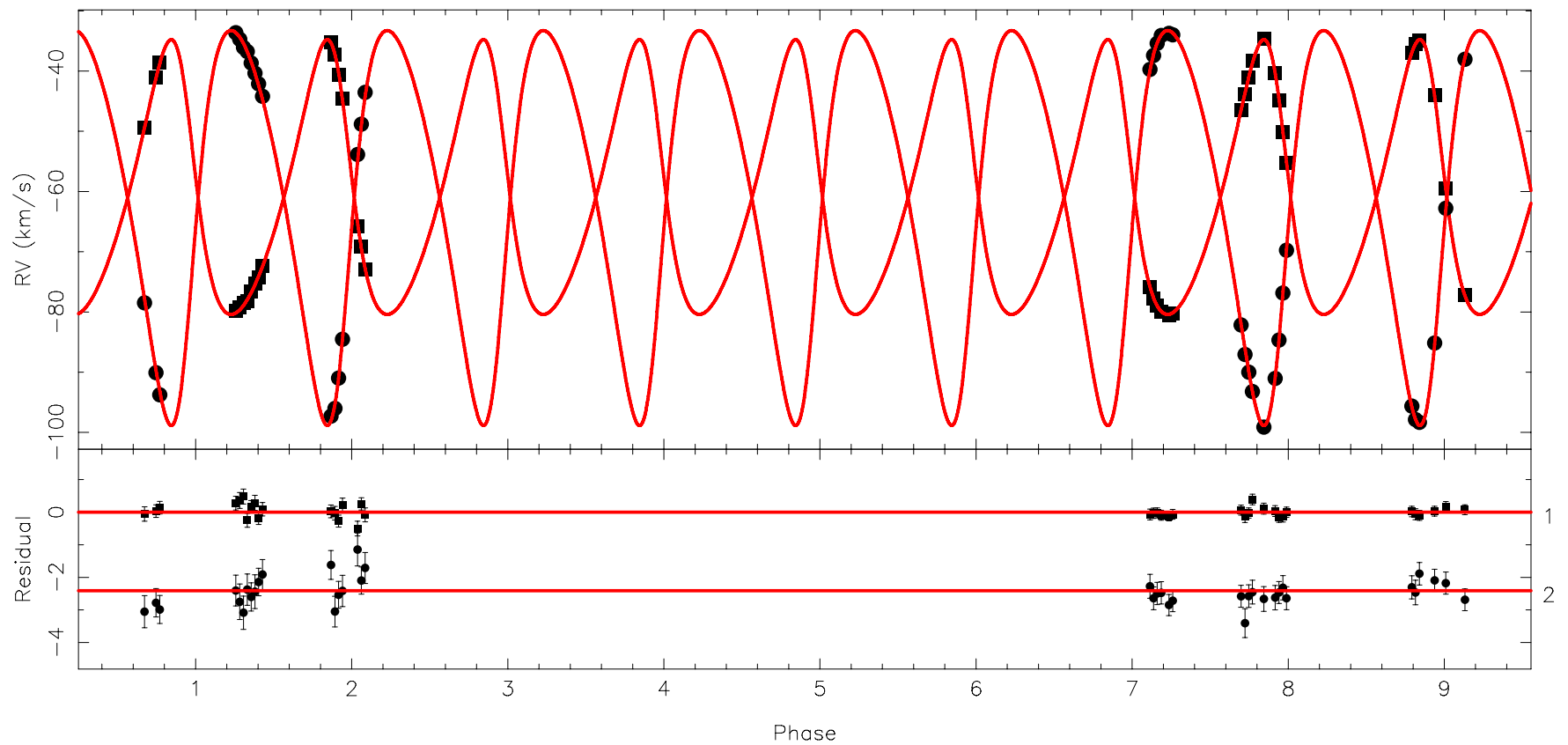

Figure 8. Radial velocity curves used in the model, with the best fit overlaid (top) and residual (bottom). The primary velocities are shown with square symbols, and the secondary velocities with round symbols. The residuals for the two stars have been offset for clarity.

(A color version of this figure is available in the online journal.)

Table 8

Derived Parameters and Uncertainties Showing the Effect of Additional Spots on the Secondary

\begin{tabular}{lccc}
\hline \hline Parameter & \multicolumn{3}{c}{ Non-eclipsed Spots } \\
\cline { 2 - 4 } & $c_{2}=0$ & $c_{2}=0.1$ & $c_{2}=0.2$ \\
\hline$J_{\text {MEarth }}$ & $0.8004 \pm 0.0066$ & $0.8157 \pm 0.0075$ & $0.8271 \pm 0.0081$ \\
$J_{\text {Bessell }}$ & $0.8259 \pm 0.0069$ & $0.8409 \pm 0.0077$ & $0.8516 \pm 0.0084$ \\
$\left(R_{1}+R_{2}\right) / a$ & $0.015551_{-0.0000044}^{+0.000042}$ & $0.015586_{-0.0000044}^{+0.000043}$ & $0.015601_{-0.0000045}^{+0.000045}$ \\
$R_{2} / R_{1}$ & $0.788 \pm 0.018$ & $0.777 \pm 0.016$ & $0.757 \pm 0.014$ \\
$\cos i$ & $0.00465_{-0.00012}^{+0.00011}$ & $0.00493_{-0.00012}^{+0.00011}$ & $0.00513_{-0.00012}^{+0.00012}$ \\
$i\left(^{\circ}\right)$ & $89.7335_{-0.0062}^{+0.0066}$ & $89.7177_{-0.0064}^{+0.0066}$ & $89.7063_{-0.0066}^{+0.0067}$ \\
$L_{2} / L_{1}$ & $0.474 \pm 0.025$ & $0.423 \pm 0.022$ & $0.360 \pm 0.017$ \\
$\left(R_{1}+R_{2}\right)\left(R_{\odot}\right)$ & $0.6815_{-0.0021}^{+0.0021}$ & $0.6829_{-0.0021}^{+0.0021}$ & $0.6834_{-0.0022}^{+0.0022}$ \\
$R_{1}\left(R_{\odot}\right)$ & $0.3814_{-0.0032}^{+0.0028}$ & $0.3844_{-0.0029}^{+0.0002}$ & $0.3892_{-0.0024}^{+0.0021}$ \\
$R_{2}\left(R_{\odot}\right)$ & $0.3001_{-0.0044}^{+0.0007}$ & $0.2985_{-0.0042}^{+0.0044}$ & $0.2942_{-0.0037}^{+0.00040}$ \\
\hline
\end{tabular}

the secondary, but we do not show results for this model since it is unable to reproduce the observed eclipse light curves, as discussed in Section 4.3.2.

Adding "non-eclipsed spots" affects the light ratio, and both component radii, slightly increasing the sum of the radii, increasing $R_{1}$, and decreasing $R_{2}$. The light ratio behaves as expected, becoming closer to unity as the primary is darkened by the addition of spots. Both $c_{2}=0.2$ models with "non-eclipsed spots" (and to some extent, the $c_{2}=0.1$ model) produce a light ratio that is marginally discrepant with the spectroscopic value (see Section 3 or Table 5), but the other models agree reasonably well within the errors.

Given the lack of clearly detected perturbations in the best quality eclipse curves, it is reasonable to favor the "non-eclipsed spots" models over the "eclipsed spots" models. Assuming the $c_{i}=0.1$ model is typical, and the $c_{i}=0$ and 0.2 models represent the likely range of values, we estimate the final system parameters and their uncertainties by combining the posterior probability distributions from all six "non-eclipsed spots" models in this section (all three values of $c_{i}$ ). These parameters and estimated uncertainties are reported in Section 4.7.

\subsection{Bandpass Mismatch}

A concern, when combining light curves taken with different instruments, is the effect of any difference in the photometric bandpasses. In the present case, where the primary and secondary eclipses were (by necessity) measured using different instruments, this predominantly affects the ratio of the eclipse depths, and thus the parameter $J$, the ratio of central surface brightnesses.

In order to determine the approximate size of the bandpass mismatch, we measured the difference in magnitude between LSPM J1112+7626 and a nearby, much bluer comparison star, at $11^{\mathrm{h}} 12^{\mathrm{m}} 12^{\mathrm{s}} .15+76^{\circ} 27^{\prime} 33^{\prime \prime} .8$ (position from 2MASS; this is the bright star seen near the upper left corner of Figure 2). The latter star has $(J-K)_{2 \text { MASS }}=0.27$ and is thus probably in the $\mathrm{F}$ spectral class. It is non-variable at the precision of the MEarth data (rms scatter $0.003 \mathrm{mag}$ ).

Our measured magnitude differences were $\Delta I($ Hankasalmi $)=$ $0.36, \Delta I($ Clay $)=0.35$, and $\Delta I($ MEarth $)=0.55$. The excellent agreement between the two $I_{\text {Bessell }}$ filters (especially in light of the observed out-of-eclipse variations of our target) indicates they are most likely an extremely good match.

Assuming a simple linear scaling, we estimate the change in $J$ corresponding to the mismatch between the two $I_{\text {Bessell }}$ filters is approximately $1 / 10$ of that between the $I_{\text {Bessell }}$ and $I_{\text {MEarth }}$ filters, or $\delta J \approx 0.003$. This is unimportant compared to the other sources of uncertainty (see Table 6).

\subsection{Limb Darkening}

\subsubsection{Differences between $I_{\text {Bessell }}$ and $I_{C}$}

We first examine the effect of assuming the limb darkening law is the same in the two $I$ filters. To do this, we compare the 


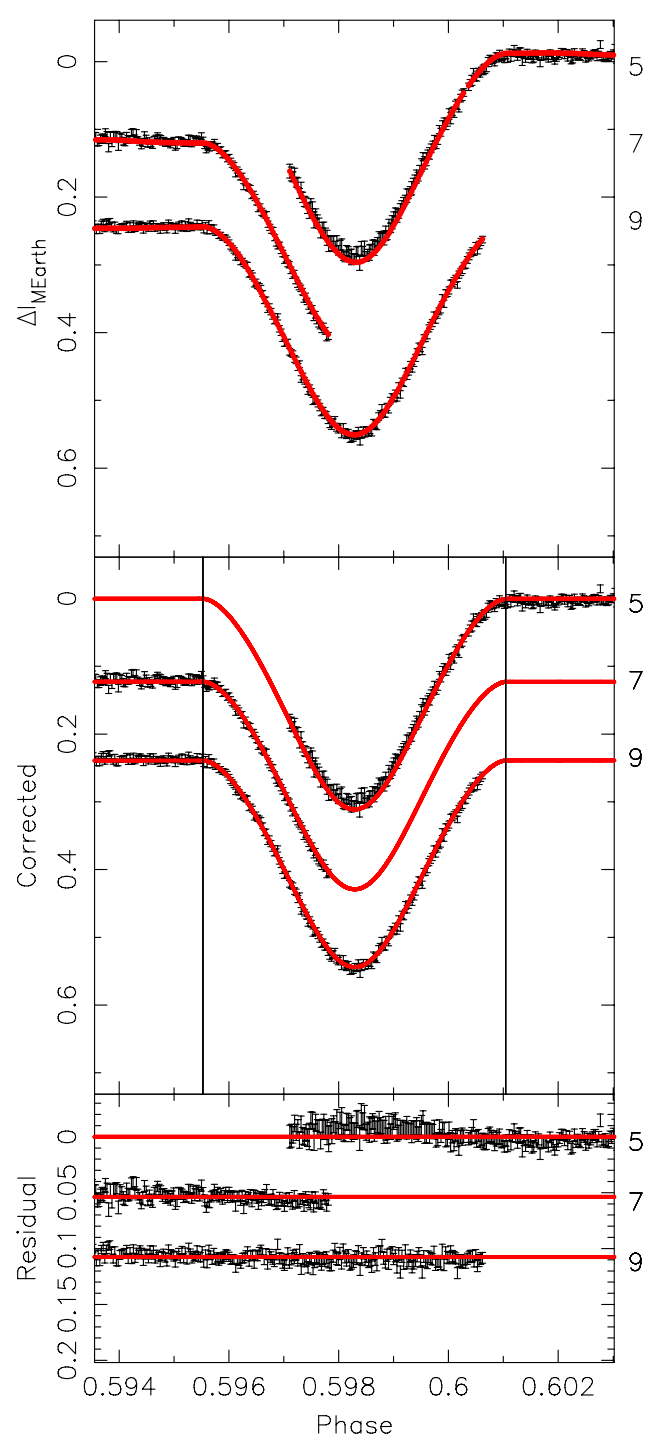

Figure 9. As Figure 6, except for the "eclipsed spots" model on the secondary, showing the deviations in the MEarth 2010 November 19 event.

(A color version of this figure is available in the online journal.)

fitting results using our baseline model to using the coefficients for the $z^{\prime}$ SDSS passband from Claret (2004), where the $I_{\text {Bessell }}$ band should lie in between these two extremes.

Using the observed colors of LSPM J1112+7626, we estimate that approximately $1 / 3$ of the difference between the $I_{C}$ and $z^{\prime}$ results is appropriate for the error introduced by our assumption of the $I_{C}$ limb darkening law in fitting the $I_{\text {Bessell }}$ data. Comparing the results for the two bands in Table 9, we find this source of error in the radii is negligible (although it does affect $J$ and $i$ at close to $1 \sigma$ ). While the radius sum error is comparable to the individual estimated uncertainties in the table, the uncertainty in this parameter for our adopted solution combining six spot configurations is larger, and the limb darkening contribution is then less important.

\subsubsection{Errors in the Atmosphere Models}

We have assumed limb darkening coefficients fixed to the theoretical values from PHOENIX model atmospheres throughout the analysis. We note that the same atmosphere models have issues reproducing the observed spectra of M-dwarfs in the optical, which raises the possibility of systematic errors in the
Table 9

Comparison of Derived Parameters and Uncertainties for $I_{C}$ and $z^{\prime}$ Limb Darkening Laws Using the "Non-eclipsed Spots" Model on the Primary

\begin{tabular}{lcc}
\hline \hline Parameter & $I_{C}$ & $z^{\prime}$ \\
\hline$J_{\text {MEarth }}$ & $0.7999 \pm 0.0064$ & $0.7829 \pm 0.0085$ \\
$J_{\text {Bessell }}$ & $0.8255 \pm 0.0066$ & $0.8073 \pm 0.0091$ \\
$\left(R_{1}+R_{2}\right) / a$ & $0.015548_{-0.00039}^{+0.00032}$ & $0.015387_{-0.00053}^{+0.000056}$ \\
$R_{2} / R_{1}$ & $0.787 \pm 0.017$ & $0.773 \pm 0.022$ \\
$\cos i$ & $0.00464_{-0.000011}^{+0.00010}$ & $0.00440_{-0.00017}^{+0.00016}$ \\
$i\left(^{\circ}\right)$ & $89.7339_{-0.0059}^{+0.0064}$ & $89.7481_{-0.0089}^{+0.0096}$ \\
$L_{2} / L_{1}$ & $0.485 \pm 0.025$ & $0.462 \pm 0.031$ \\
$\left(R_{1}+R_{2}\right)\left(R_{\odot}\right)$ & $0.6814_{-0.0020}^{+0.0020}$ & $0.6744_{-0.0027}^{+0.0025}$ \\
$R_{1}\left(R_{\odot}\right)$ & $0.3815_{-0.0030}^{+0.0028}$ & $0.3807_{-0.0033}^{+0.0033}$ \\
$R_{2}\left(R_{\odot}\right)$ & $0.2999_{-0.0043}^{+0.0044}$ & $0.2938_{-0.0055}^{+0.0059}$ \\
\hline
\end{tabular}

limb darkening law. In the literature, this is conventionally addressed by analyzing photometry in multiple passbands, which is sensitive to differences in the limb darkening as a function of wavelength. We do not have multi-band photometry, but note this could be an important area for future work.

It has long been recognized (e.g., Nelson \& Davis 1972, and references therein) that changes in the radius of the eclipsed star mimic changes in the limb darkening. In a system with nearequal stars such as the present, it is reasonable to expect any systematic error in the limb darkening from the models should affect both stars similarly, and thus to first order, the dominant effect will be on the sum of the radii (and the inclination, but any uncertainties here have negligible effect on the final parameters).

We verified this using simulations where the square root limb darkening coefficients were varied, finding a strong correlation between $\left(R_{1}+R_{2}\right) / a$ and the integral of the specific intensity over the stellar disk, which is $1-u / 3-u^{\prime} / 5$ for the square root law (it is also correlated to a lesser extent with $J$ and $\cos i$ ). This quantity is the normalization term in the eclipse depths in the photometric model, so it is not surprising that it should directly influence the quantities derived from the absolute eclipse depth. This is predominantly a concern for the interpretation of the sum of the radii: we find even quite large changes in the limb darkening law do not significantly alter the individual radii compared to their uncertainties in our adopted model.

\subsection{Third Light}

We now examine our assumption of no third light. While the proper motion evidence (see Figure 2) argues it is unlikely there are any background stars contributing at a significant level in the photometric aperture, the relatively low proper motion of our target does not allow this possibility to be completely eliminated given the limited angular resolution of the first epoch imaging data. Common proper motion companions are also permitted at intermediate separations below the angular resolution of the SDSS data, but still in wide enough orbits or with mass ratios much less than unity, where they would not be detected by the presence of additional lines in the spectra or radial velocity drift over the approximately one year baseline available.

Therefore, to examine any constraints on third light which can be placed directly from the light curves, and the effect on the derived parameters, we ran an additional set of Monte Carlo simulations using the basic "non-eclipsed spots" model on the primary discussed in Section 4.3.2 and Table 6, allowing $L_{3}$ to vary. For simplicity, a uniform prior was assumed. 
Table 10

Adopted Physical Parameters and Uncertainties, Combining Six Spot Configurations

\begin{tabular}{|c|c|c|}
\hline \multirow[t]{2}{*}{ Parameter } & \multicolumn{2}{|c|}{ Value } \\
\hline & $\mathrm{C}^{2} 8^{\mathrm{a}}$ & L96 ${ }^{\mathrm{a}}$ \\
\hline$M_{1}\left(M_{\odot}\right)$ & \multicolumn{2}{|c|}{$0.3946 \pm 0.0023$} \\
\hline$M_{2}\left(M_{\odot}\right)$ & \multicolumn{2}{|c|}{$0.2745 \pm 0.0012$} \\
\hline$\left(R_{1}+R_{2}\right)\left(R_{\odot}\right)$ & \multicolumn{2}{|c|}{$0.6834_{-0.0028}^{+0.0046}$} \\
\hline$R_{1}\left(R_{\odot}\right)$ & \multicolumn{2}{|c|}{$0.3860_{-0.0052}^{+0.0055}$} \\
\hline$R_{2}\left(R_{\odot}\right)$ & \multicolumn{2}{|c|}{$0.2978_{-0.0046}^{+0.0049}$} \\
\hline $\log g_{1}$ & \multicolumn{2}{|c|}{$4.861 \pm 0.012$} \\
\hline $\log g_{2}$ & \multicolumn{2}{|c|}{$4.929 \pm 0.014$} \\
\hline$T_{\mathrm{eff}, 1}^{\mathrm{a}}(\mathrm{K})$ & $3061 \pm 162$ & $3191 \pm 164$ \\
\hline$T_{\mathrm{eff}, 2^{\mathrm{a}}}(\mathrm{K})$ & $2952 \pm 163$ & $3079 \pm 166$ \\
\hline$T_{\mathrm{eff}, 2} / T_{\mathrm{eff}, 1}$ & $0.959_{-0.025}^{+0.049}$ & $0.960_{-0.025}^{+0.048}$ \\
\hline$L_{\text {bol,1 }}\left(L_{\odot}\right)$ & $0.0119 \pm 0.0025$ & $0.0141 \pm 0.0028$ \\
\hline$L_{\text {bol, } 2}\left(L_{\odot}\right)$ & $0.0061 \pm 0.0014$ & $0.0073 \pm 0.0016$ \\
\hline$(m-M)$ & $3.40 \pm 0.22$ & $3.53 \pm 0.22$ \\
\hline$d(\mathrm{pc})$ & $48.1 \pm 5.0$ & $51.0 \pm 5.2$ \\
\hline$U^{\mathrm{b}}\left(\mathrm{km} \mathrm{s}^{-1}\right)$ & $56.1 \pm 3.1$ & $57.6 \pm 3.2$ \\
\hline$V\left(\mathrm{~km} \mathrm{~s}^{-1}\right)$ & $-44.4 \pm 1.7$ & $-44.8 \pm 1.8$ \\
\hline$W\left(\mathrm{~km} \mathrm{~s}^{-1}\right)$ & $-10.8 \pm 3.2$ & $-9.1 \pm 3.4$ \\
\hline
\end{tabular}

Notes.

${ }^{\text {a }}$ Effective temperatures and bolometric corrections used. C08: Casagrande et al. (2008); L96: Leggett et al. (1996). A $150 \mathrm{~K}$ systematic uncertainty was assumed for the effective temperatures, and the color equations given in the 2MASS explanatory supplement were used to convert the observed $J H K$ photometry to the CIT system when using the L96 tabulation.

b Adopting the definition of positive $U$ toward the Galactic center. Calculated using the method of Johnson \& Soderblom (1987).

We find $L_{3}<0.029$ at $95 \%$ confidence. As discussed by Nelson \& Davis (1972), the dominant parameter mimic is between third light and inclination, and our results confirm this, finding the uncertainties in $\cos i$ and the central surface brightness ratio parameters were slightly inflated. The other light curve parameters and radii derived from these chains are indistinguishable within the uncertainties from the results where $L_{3}$ was not varied.

\subsection{Summary and Adopted System Parameters}

Of the sources of uncertainty we have considered, spots dominate. Since the stars hosting the spots and the spot configuration are mostly unknown, we adopt the union of the six spotted solutions discussed in Section 4.3.3, giving them equal weight. The final parameter estimates and their uncertainties are reported in Table 10, derived from the posterior samples produced by the Monte Carlo simulations, adopting the median and $68.3 \%$ central confidence intervals as the central value and uncertainty (see Section 4.2).

We compute $U V W$ space motions for LSPM J1112+7626 using the method of Johnson \& Soderblom (1987), with the distance from the EB solution, $\gamma$ velocity from Table 6 , and position and proper motions from Table 3 . We find this object is in the old Galactic disk population, following the method and definitions in Leggett (1992).

For very precise work, even matters as seemingly trivial as physical constants can be important, so we briefly discuss our assumptions in this regard. We adopt the 2009 IAU values of $G M_{\odot}$ and the astronomical unit (see the Astronomical Almanac 2011), and follow Cox (2000) in adopting the value of the solar photospheric radius from Brown \& ChristensenDalsgaard (1998). ${ }^{11}$ For the solar effective temperature, we use the value of $5781 \mathrm{~K}$ from Bessell et al. (1998), which is based on measurements of the solar constant by Duncan et al. (1982).

The final constant, the solar bolometric absolute magnitude, is a more thorny issue, as discussed by Bessell et al. (1998), and extensively by Torres (2010). When using tables of bolometric corrections, it is vital to adopt the consistent value of this quantity in accordance with the table. We therefore use the appropriate values in each case in the following section.

\subsection{Effective Temperatures, Bolometric Luminosities, and Distance}

In order to estimate effective temperatures (and thus, bolometric luminosities, and the distance), an external estimate of the effective temperature of one of the binary components is required, in addition to bolometric corrections. For M-dwarfs, there are large systematic uncertainties in the effective temperature scale (e.g., Allard et al. 1997; Luhman \& Rieke 1998), with disagreement at the few hundred degrees Kelvin level among different authors. Many of the early difficulties were due to the lack of model atmospheres (which are usually needed to integrate the full spectral energy distribution from the available measurements to obtain $L_{\mathrm{bol}}$ ), and significant improvement on this front was made in the 1990s. The availability of a much larger sample of angular diameter measurements from interferometry should further improve the situation as this provides a much more direct method to estimate $T_{\text {eff }}$ for single stars, but there are relatively few temperature scales available at the present time using this information.

We show results from two different inversions to illustrate the typical range of parameters. Both scales we use were derived with model atmospheres rather than blackbodies, and in both cases we use the measured $I_{C}-J_{2 \text { MASS }}, I_{C}-H_{2 \text { MASs }}$, and $I_{C}-K_{2 \text { MASS }}$ colors from Table 3 in conjunction with the component radii and $I_{C}$-band light ratio from the adopted EB solution. The results from these different pairings of bandpasses were found to be consistent within the uncertainties, so we report the union of the results.

The two sets of effective temperature and bolometric corrections adopted were from Casagrande et al. (2008), which is a recent determination using interferometric angular diameter measurements to derive $T_{\text {eff }}$, and from Leggett et al. (1996), which is the basis for several recent works and tabulations of the effective temperature scale, including Luhman \& Rieke (1998), Luhman (1999), and Kraus \& Hillenbrand (2007).

For the Leggett et al. (1996) scale, we fit polynomials to their tabulated data, omitting three objects these authors found to be metal poor from our fits: Gl 129, LHS 343, and LHS 377. We follow Leggett et al. (1996) in adopting a systematic uncertainty of $150 \mathrm{~K}$, which appears to be consistent with the differences we find between the two determinations.

Our results for LSPM J1112+7626 are reported in Table 10.

\section{DISCUSSION}

We now compare our results for LSPM J1112+7626 with theoretical models from Baraffe et al. (1998) and other

\footnotetext{
11 A useful compilation of solar data may be found in "Basic Astronomical Data for the Sun," by Eric Mamajek (University of Rochester, NY, USA), available at http://www.pas.rochester.edu/ emamajek/sun.txt.
} 
Table 11

Detached, Double-lined, Double-eclipsing Main-sequence EB Components below $0.4 M_{\odot}{ }^{\mathrm{a}}$

\begin{tabular}{|c|c|c|c|c|c|c|}
\hline Name & $\begin{array}{l}\text { Period } \\
\text { (days) }\end{array}$ & $\begin{array}{c}M \\
\left(M_{\odot}\right)\end{array}$ & $\begin{array}{c}R \\
\left(R_{\odot}\right) \\
\end{array}$ & $\begin{array}{l}T_{\text {eff }} \\
(\mathrm{K})\end{array}$ & {$[\mathrm{M} / \mathrm{H}]$} & Source $^{b}$ \\
\hline SDSS-MEB-1 A & 0.407 & $0.272 \pm 0.020$ & $0.268 \pm 0.0090$ & $3320 \pm 130$ & $\ldots$ & 1 \\
\hline SDSS-MEB-1 B & & $0.240 \pm 0.022$ & $0.248 \pm 0.0084$ & $3300 \pm 130$ & & \\
\hline GJ $3236 \mathrm{~A}^{\mathrm{c}}$ & 0.771 & $0.376 \pm 0.016$ & $0.3795 \pm 0.0084$ & $3312 \pm 110$ & $\ldots$ & 2 \\
\hline GJ $3236 \mathrm{~B}^{\mathrm{c}}$ & & $0.281 \pm 0.015$ & $0.300 \pm 0.015$ & $3242 \pm 108$ & & \\
\hline CM Dra A & 1.27 & $0.2310 \pm 0.0009$ & $0.2534 \pm 0.0019$ & $3130 \pm 70$ & {$[-1,-0.6]$} & 3 \\
\hline CM Dra B & & $0.2141 \pm 0.0010$ & $0.2396 \pm 0.0015$ & $3120 \pm 70$ & & \\
\hline LP 133-373 A & 1.63 & $0.340 \pm 0.014$ & $0.33 \pm 0.02$ & $3058 \pm 195$ & $\ldots$ & 4 \\
\hline LP 133-373 B & & Same & Same & $3144 \pm 206$ & $\ldots$ & \\
\hline MG1-2056316 B & 1.72 & $0.382 \pm 0.001$ & $0.374 \pm 0.002 \pm 0.002$ & $3320 \pm 180$ & $\ldots$ & 5 \\
\hline KOI $126 \mathrm{~B}^{\mathrm{d}}$ & 1.77 & $0.2413 \pm 0.0030$ & $0.2543 \pm 0.0014$ & $\ldots$ & $+0.15 \pm 0.08$ & 6 \\
\hline KOI $126 \mathrm{C}^{\mathrm{d}}$ & & $0.2127 \pm 0.0026$ & $0.2318 \pm 0.0013$ & $\ldots$ & & \\
\hline CCDM J04404+3127 B, $\mathrm{C}^{\mathrm{e}}$ & 2.05 & $\ldots$ & $\ldots$ & $\ldots$ & $\ldots$ & 7 \\
\hline CU Cnc B & 2.77 & $0.3980 \pm 0.0014$ & $0.3908 \pm 0.0094$ & $3125 \pm 150$ & $\ldots$ & 8 \\
\hline 1RXS J154727.5+450803 A & 3.55 & $0.2576 \pm 0.0085$ & $0.2895 \pm 0.0068$ & $\ldots$ & $\ldots$ & 9 \\
\hline 1RXS J154727.5+450803 B & & $0.2585 \pm 0.0080$ & Same & $\ldots$ & & \\
\hline
\end{tabular}

Notes.

a This mass criterion has been applied to keep the number of objects in the table and plots manageable, and is not intended to necessarily be physically meaningful beyond being appropriate for comparison to the present system.

${ }^{b}$ References. (1) Blake et al. 2008; (2) Irwin et al. 2009b; (3) Morales et al. 2009; (4) Vaccaro et al. 2007; (5) Kraus et al. 2011; (6) Carter et al. 2011; (7) Shkolnik et al. 2010; (8) Ribas 2003; Delfosse et al. 1999; (9) Hartman et al. 2011.

${ }^{c}$ Parameters determined giving equal weight to all three models, following Hartman et al. (2011).

${ }^{\mathrm{d}}$ While not double-lined, this object is a special case as it is still possible to solve for the masses and radii of both M-dwarfs independent of M-dwarf models. The period given is that for the inner M-dwarf binary as this is presumably the appropriate one for estimation of activity levels.

${ }^{\mathrm{e}}$ Full solution not available.

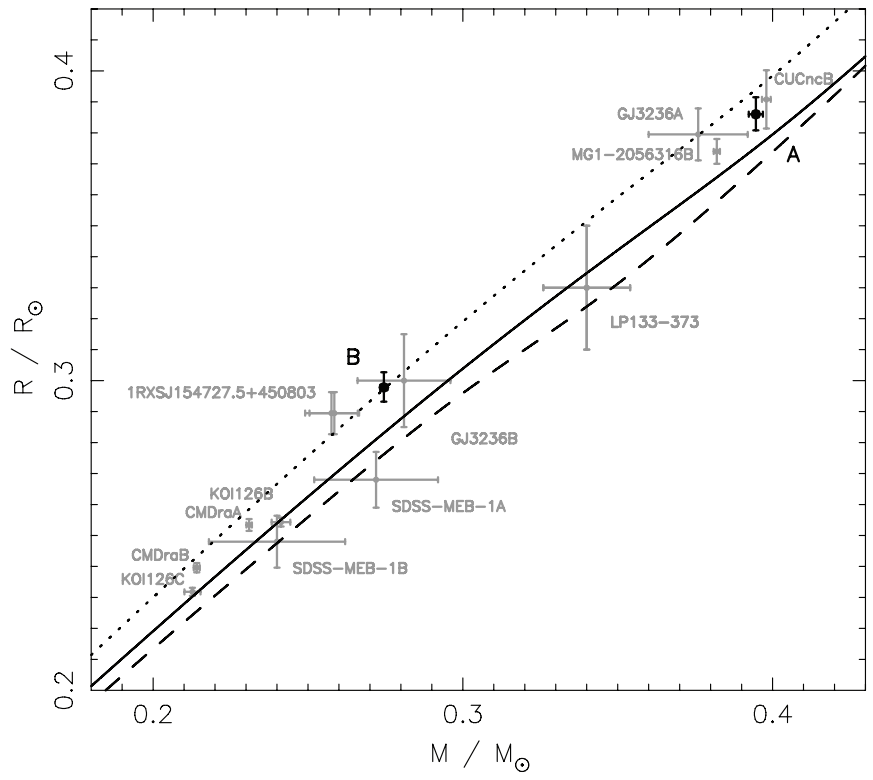

Figure 10. Mass-radius relation for components of detached, double-lined, double-eclipsing binary systems below $0.4 M_{\odot}$. LSPM J1112+7626 is shown as bold, black points, and the literature systems from Table 11 are shown in gray, excluding CCDM J04404+3127B,C, which does not have a full solution available at present, but including KOI 126. The lines show stellar evolution models from Baraffe et al. (1998) for $10 \mathrm{Gyr}$ age, $[\mathrm{M} / \mathrm{H}]=0$ (solid line), $[\mathrm{M} / \mathrm{H}]=-0.5$ (dashed line), and the $[\mathrm{M} / \mathrm{H}]=0$ model with the radius inflated by $5 \%$, corresponding to $\rho=1.05$ (dotted line).

literature objects with shorter periods summarized in Table 11. We first show the conventional mass-radius diagram in Figure 10 , and then the corresponding mass-effective-temperature $\left(T_{\text {eff }}\right)$ diagram in Figure 11.

We also perform a quantitative comparison with the same models. We follow Torres (2007) in defining a parameter equal

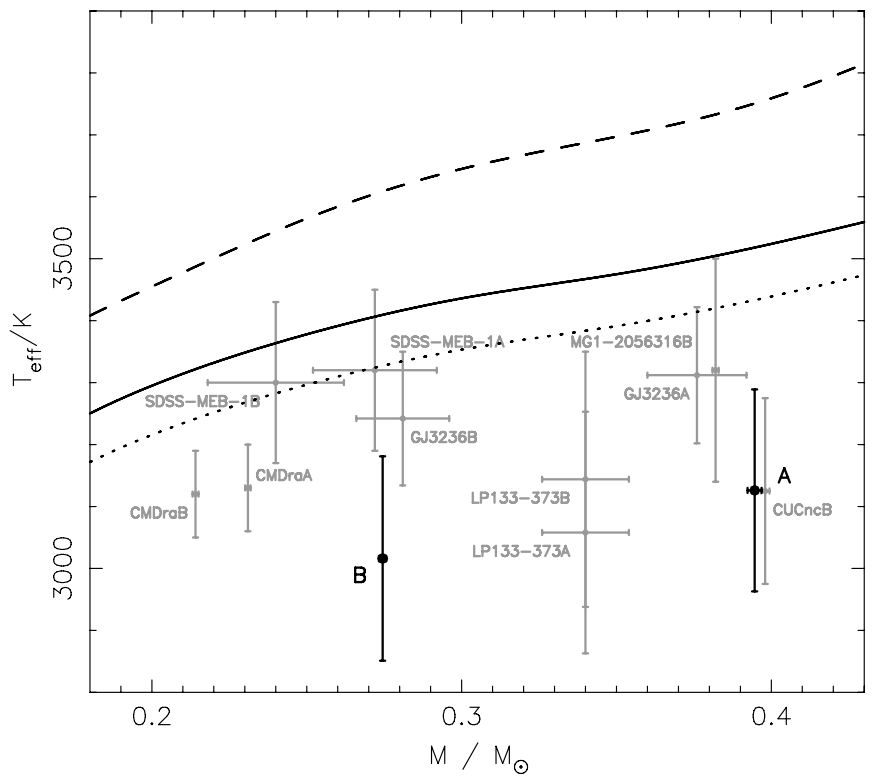

Figure 11. Mass-effective-temperature relation for components of detached, double-lined, double-eclipsing binary systems below $0.4 M_{\odot}$. Lines and points as Figure 10. KOI 126B,C and 1RXS J154727.5+450803 do not have reported effective temperatures, so they do not appear on this diagram. The dotted line shows the prediction from assuming $\rho=1.05$ and that the bolometric luminosity is preserved.

to the ratio of the observed radius to that predicted by the models given the observed mass, although we denote this parameter by $\rho$ instead, to avoid any potential confusion with the $\beta$ parameter defined by Chabrier et al. (2007) and used by Morales et al. (2010), which is not the same.

As argued in Torres (2007), there is evidence that the bolometric luminosities from the models are not seriously in error. If this is the case, a corresponding factor of $\rho^{-1 / 2}$ must be 
Table 12

Quantitative Mass-Radius Comparison of LSPM J1112+7626 with Models

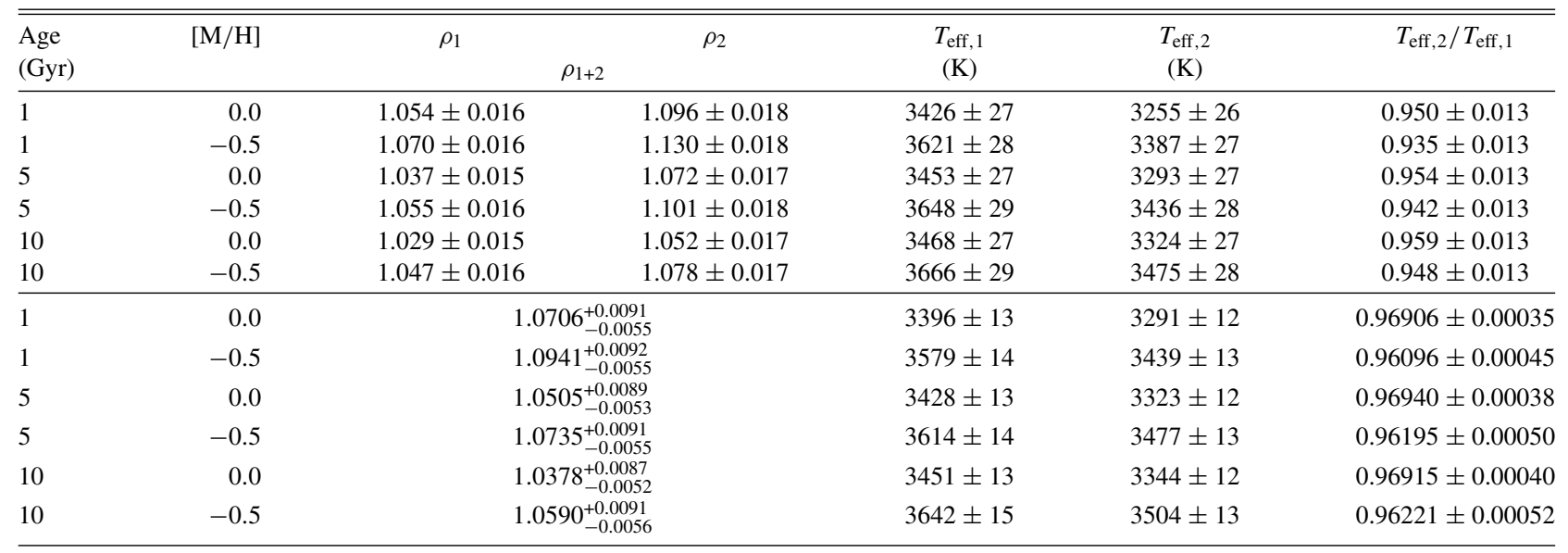

applied to the effective temperatures from the models when inflating the radii, in order to preserve the bolometric luminosity. We show results for this scenario in the tables and plots. Note, however, that the bolometric luminosity is not preserved in several recent theoretical works (Chabrier et al. 2007; Morales et al. 2010; MacDonald \& Mullan 2011), so the temperatures derived from this procedure should not be considered definitive.

As is often the case in EBs, while the individual masses of the objects are well-determined from the radial velocities, the sum of the radii is much better determined than the individual radii, modulo limb darkening uncertainties as discussed in Section 4.5. We therefore compute, in addition to the $\rho$ values for the individual stars, a "composite" value comparing the modelpredicted radius sum given the individual observed masses, to the measured radius sum. This quantity, which we call $\rho_{1+2}$, has much smaller formal uncertainties than the individual $\rho_{1}$ and $\rho_{2}$ values, and measures essentially a "weighted average inflation" for the whole system. These quantities, and the corresponding effective temperatures predicted from preserving the bolometric luminosity, are given for a range of assumptions for the age and metallicity in Table 12. While we caution overinterpretation of the $\rho_{1+2}$ results due to limb darkening uncertainty, the individual values should be much less prone to this problem.

\subsection{Mass-Radius Comparison}

We first limit our comparisons to the mass-radius plane. If the metallicity is solar, the sum of radii is inflated at the $7 \sigma$ level compared to the models, even for $10 \mathrm{Gyr}$ age, which produces the largest model radii. The discrepancies are not as significant in the individual radii, with the primary $2 \sigma$ and the secondary $3 \sigma$ larger than the model predictions. We also note that the "eclipsed spots" model on the secondary, which we discarded in Section 4.3, does not solve the radius discrepancy, having a secondary radius (and sum of radii) even further from the model predictions.

Youth is unlikely as an explanation given the old disk kinematics of our target. We estimate an age of approximately $120 \mathrm{Myr}$ at solar metallicity would be needed to bring the radius sum into agreement with the models, yet such objects should also rotate extremely rapidly and exhibit strong $\mathrm{H} \alpha$ emission (e.g., Terndrup et al. 2000; Hartman et al. 2010, and references therein), neither of which are seen.

Metallicity may provide a viable explanation for the observed radii given the present uncertainties (both in the observations and the models, where for the latter it is possible the effect of metallicity on radius is underpredicted; see Section 1 and López-Morales 2007), although the object would most likely need to be extremely metal rich. This would place it in the tail of the metallicity distribution for the old Galactic disk population, which is likely to peak around $[\mathrm{M} / \mathrm{H}] \approx-0.5$ (e.g., Leggett 1992, and references therein).

Given that metallicity has difficulty reproducing radii in other well-observed systems (see Section 1) and the kinematic evidence, we proceed to examine other possible causes of the inflated radii.

It is tempting to suggest that the inflation in LSPM $\mathrm{J} 1112+7626$ results from elevated activity levels in one or both components. While the lack of $\mathrm{H} \alpha$ emission, unless this is rendered undetectable by the limited signal-to-noise ratio of our spectra, may be a difficulty for this scenario to explain, it should be noted that more modest levels of activity can result in weakened $\mathrm{H} \alpha$ emission, or even absorption, but may still produce sufficient spot coverage to explain the inflation.

This system straddles (in mass) the full convection limit, where a substantial increase in the observed activity lifetimes for M-dwarfs occurs (e.g., West et al. 2008). This would argue that the secondary is more likely to be active and thus the source of the inflation. The secondary lines in the spectrum are also more difficult to observe due to its lower luminosity, which would make an $\mathrm{H} \alpha$ line easier to hide in the noise. Identification of the star hosting the out-of-eclipse modulations may shed additional light on which of the components might be responsible for the inflation.

As discussed by Morales et al. (2010), the radius inflation inferred from EB analyses often implies extremely high spot filling factors. We estimate that $\rho=1.05$ corresponds to $\beta=$ 0.1 . Assuming a spot temperature contrast of $T_{\text {eff, spot }} / T_{\text {eff,phot }}=$ 0.9 , this would require a filling factor of approximately $30 \%$. This is much larger than needed to produce the observed outof-eclipse modulation and would mean there is a substantial longitudinally symmetric spot component, if the inflation is indeed caused by spots. We showed in Section 4.3.3 that such a level of spots does slightly modify the component radii if they are not eclipsed, reducing $R_{2}$ and increasing $R_{1}$. In practice, this may slightly reduce the required filling factor depending on where the spots are located, but unless the spot coverage is much larger than we assumed, the systematic errors in the radii resulting from such spots are still insufficient to explain the observations without invoking inflation. 
Finally, on suggestion of the referee, we note that this object may indicate the need to revisit the equation of state for very low mass stars, which was discussed as a possibility to explain the radius inflation before the activity hypothesis gained prevalence (e.g., Lopez-Morales 2004; Torres \& Ribas 2002). The equation of state was a significant source of difficulty in early work attempting to model very low mass stars (Chabrier \& Baraffe 1995), and although substantial progress on this front has been made (see the reviews by Chabrier \& Baraffe 2000; Chabrier et al. 2005), it is still an open question.

\subsection{Mass- $T_{\text {eff }}$ Comparison}

Comparisons in only the mass-radius plane ignore important information contained in the effective temperatures. The correct physical model must explain all of the observations simultaneously, so we now proceed to examine the temperatures. This comparison is more problematic than in mass-radius for a number of reasons. The main observational issues are the difficulty of constraining effective temperatures (see Section 4.8) and metallicities for the EBs.

As noted in Section 1, unlike the radius, the effective temperature predicted from models does depend quite strongly on metallicity, because the bolometric luminosity is a function of metallicity. As the metallicity is decreased, the model bolometric luminosity and effective temperature increase, and the radius decreases by a small amount. While metallicity does complicate the interpretation of the mass $-T_{\text {eff }}$ diagram, this is a key argument against metallicity as the explanation for all of the inflated radii in the EB sample, as discussed already in Section 1. In this regard, CM Dra is puzzling as several authors have claimed this object is metal poor, which would further exacerbate the effective temperature discrepancy with the models.

Given the present uncertainties, it is not clear if the effective temperature difference between LSPM J1112+7626 and the models is significant. However, it does lie at the low-temperature end of the EB results, and this lends support to the hypothesis that this object is more metal rich than the other EBs.

\subsection{Future Work}

It is clear that LSPM J1112+7626 offers one of the best prospects to observationally test the causes of inflated radii in EBs, but further observations are needed. The most fruitful avenues would be to pursue a determination of the metallicity, improvement of the system parameters with a particular focus to investigating potential systematics in the radii (e.g., due to spots), and constraining the activity levels in the components by independent means (for example, X-ray emission, or the CaII $\mathrm{H}$ and $\mathrm{K}$ lines).

While we have mentioned the non-synchronized spin and orbit, we were unable to take full advantage of this property with the available observational data. As shown in Section 4.3, the effect of spots should be larger on the primary eclipse, so we advocate an intensive campaign on these events, preferably to obtain multiple, complete eclipses with the same telescope and detector system at a range of rotational phases and in multiple wavelengths. The primary eclipses are observable from Europe and Scandinavia during the winter 2011-2012 season, where excellent observational facilities are available. We remind observers that good-quality out-of-eclipse monitoring will be important for the interpretation of the eclipses themselves, and the spot distributions are likely to evolve (indeed, Figure 7 indicates that we may have already seen this in the existing data), so the monitoring must be contemporaneous, and preferably also taken at multiple wavelengths to constrain the spot temperatures.

The MEarth team gratefully acknowledges funding from the David and Lucile Packard Fellowship for Science and Engineering (awarded to D.C.) and the National Science Foundation (NSF) under grant number AST-0807690. S.N.Q., D.W.L., and G.A.E. acknowledge partial support from the NASA Kepler mission under cooperative agreement NCC2-1390, and G.T. acknowledges partial support from NSF grant AST-1007992. We thank Allyson Bieryla for assistance with the Clay telescope observations, David Kipping for discussions of appropriate priors for use in Monte Carlo simulations, Eric Mamajek for compiling and maintaining the "Basic Astronomical Data for the Sun" page, Todd Vaccaro for clarification of the parameters of LP 133-373, Andrew West for discussions of M-dwarf activity, and Edo Berger and the 2011 Harvard Astronomy 100 undergraduate class for assistance with the FAST observations. The anonymous referee is thanked for a prompt and helpful report that improved the manuscript. The MEarth team is greatly indebted to the staff at the Fred Lawrence Whipple Observatory for their efforts in construction and maintenance of the facility, and thank Wayne Peters, Ted Groner, Karen Erdman-Myres, Grace Alegria, Rodger Harris, Bob Hutchins, Dave Martina, Dennis Jankovsky, and Tom Welsh for their support.

This research has made extensive use of data products from the Two Micron All Sky Survey, which is a joint project of the University of Massachusetts and the Infrared Processing and Analysis Center/California Institute of Technology, funded by NASA and the NSF, NASA's Astrophysics Data System (ADS) bibliographic services, and the SIMBAD database, operated at CDS, Strasbourg, France. The Digitized Sky Surveys were produced at the Space Telescope Science Institute under U.S. Government grant NAG W-2166. The images of these surveys are based on photographic data obtained using the Oschin Schmidt Telescope on Palomar Mountain and the UK Schmidt Telescope. The plates were processed into the present compressed digital form with the permission of these institutions.

Funding for the SDSS and SDSS-II has been provided by the Alfred P. Sloan Foundation, the Participating Institutions, the National Science Foundation, the U.S. Department of Energy, the National Aeronautics and Space Administration, the Japanese Monbukagakusho, the Max Planck Society, and the Higher Education Funding Council for England. The SDSS Web site is http://www.sdss.org/. The SDSS is managed by the Astrophysical Research Consortium for the Participating Institutions. The Participating Institutions are the American Museum of Natural History, Astrophysical Institute Potsdam, University of Basel, University of Cambridge, Case Western Reserve University, University of Chicago, Drexel University, Fermilab, the Institute for Advanced Study, the Japan Participation Group, Johns Hopkins University, the Joint Institute for Nuclear Astrophysics, the Kavli Institute for Particle Astrophysics and Cosmology, the Korean Scientist Group, the Chinese Academy of Sciences (LAMOST), Los Alamos National Laboratory, the Max-Planck-Institute for Astronomy (MPIA), the Max-PlanckInstitute for Astrophysics (MPA), New Mexico State University, Ohio State University, University of Pittsburgh, University of Portsmouth, Princeton University, the United States Naval Observatory, and the University of Washington.

Facilities: FLWO:1.5m (FAST, TRES) 


\section{REFERENCES}

Abazajian, K. N., Adelman-McCarthy, J. K., Agüeros, M. A., et al. 2009, ApJS, 182,543

Allard, F., Hauschildt, P. H., Alexander, D. R., \& Starrfield, S. 1997, ARA\&A, 35,137

Andersen, J. 1991, A\&AR, 3, 91

Baraffe, I., Chabrier, G., Allard, F., \& Hauschildt, P. H. 1998, A\&A, 337, 403

Barnard, E. E. 1916, AJ, 29, 181

Barnes, J. R., \& Collier Cameron, A. 2001, MNRAS, 326, 950

Barnes, J. R., James, D. J., \& Collier Cameron, A. 2004, MNRAS, 352, 589

Barnes, J. R., Jeffers, S. V., \& Jones, H. R. A. 2011, MNRAS, 412, 1599

Bean, J. L., Sneden, C., Hauschildt, P. H., Johns-Krull, C. M., \& Benedict, G. F. 2006, ApJ, 652, 1604

Benedict, G. F., McArthur, B., Nelan, E., et al. 1998, AJ, 116, 429

Berger, D. H., Gies, D. R., McAlister, H. A., et al. 2006, ApJ, 644, 475

Berta, Z. K., Charbonneau, D., Bean, J., et al. 2011, ApJ, 736, 12

Bessel, M. S. 1990a, A\&AS, 83, 357

Bessell, M. S. 1990b, PASP, 102, 1181

Bessell, M. S., Castelli, F., \& Plez, B. 1998, A\&A, 333, 231

Blake, C. H., Torres, G., Bloom, J. S., \& Gaudi, B. S. 2008, ApJ, 684, 635

Bonfils, X., Delfosse, X., Udry, S., et al. 2005, A\&A, 442, 635

Boyajian, T. S., von Braun, K., van Belle, G., et al. 2011, in ASP Conf. Ser., Cool Stars, Stellar Systems and the Sun XVI, ed. C. Johns-Krull, A. West, \& M. Browning, (San Francisco, CA: ASP), in press (arXiv:1012.0542)

Brown, T. M., \& Christensen-Dalsgaard, J. 1998, ApJ, 500, L195

Buchhave, L. A., Bakos, G. Á., Hartman, J. D., et al. 2010, ApJ, 720, 1118

Carney, B. W., Latham, D. W., \& Laird, J. B. 1989, AJ, 97, 423

Carter, J. A., Fabrycky, D. C., Ragozzine, D., et al. 2011, Science, 331, 562

Casagrande, L., Flynn, C., \& Bessell, M. 2008, MNRAS, 389, 585

Chabrier, G., \& Baraffe, I. 1995, ApJ, 451, L29

Chabrier, G., \& Baraffe, I. 1997, A\&A, 327, 1039

Chabrier, G., \& Baraffe, I. 2000, ARA\&A, 38, 337

Chabrier, G., Baraffe, I., Allard, F., \& Hauschildt, P. H. 2005, in ASP Conf. Ser., Resolved Stellar Populations, ed. D. Valls-Gabaud \& M. Chavez (San Francisco, CA: ASP), in press (arXiv:astro-ph/0509798)

Chabrier, G., Gallardo, J., \& Baraffe, I. 2007, A\&A, 472, L17

Claret, A. 2000, A\&A, 363, 1081

Claret, A. 2004, A\&A, 428, 1001

Coughlin, J. L., López-Morales, M., Harrison, T. E., Ule, N., \& Hoffman, D. I. 2011, AJ, 141, 78

Covey, K. R., Ivezić, Ž., Schlegel, D., et al. 2007, AJ, 134, 2398

Cox, A. N. (ed.) 2000, Allen's Astrophysical Quantities (4th ed.; New York: AIP)

Delfosse, X., Forveille, T., Ségransan, D., et al. 2000, A\&A, 364, 217

Delfosse, X., Forveille, T., Udry, S., et al. 1999, A\&A, 350, L39

Demory, B.-O., Ségransan, D., Forveille, T., et al. 2009, A\&A, 505, 205

Devor, J., Charbonneau, D., Torres, G., et al. 2008, ApJ, 687, 1253

Diaz-Cordoves, J., \& Gimenez, A. 1992, A\&A, 259, 227

Doi, M., Tanaka, M., Fukugita, M., et al. 2010, AJ, 139, 1628

Duncan, C. H., Willson, R. C., Kendall, J. M., Harrison, R. G., \& Hickey, J. R. 1982, Sol. Energy, 28, 385

Eggen, O. J., \& Sandage, A. 1967, ApJ, 148, 911

Fabricant, D., Cheimets, P., Caldwell, N., \& Geary, J. 1998, PASP, 110, 79

Ford, E. B. 2005, AJ, 129, 1706

Ford, E. B. 2006, ApJ, 642, 505

Gizis, J. E. 1997, AJ, 113, 806

Gizis, J. E., Reid, I. N., \& Hawley, S. L. 2002, AJ, 123, 3356

Gregory, P. C. 2005, ApJ, 631, 1198

Griffin, R. F., Radford, G. A., Harmer, D. L., \& Stickland, D. J. 1976, The Observatory, 96, 153

Haario, P. C., Saksman, E., \& Tamminen, J. 2001, Bernoulli, 7, 223

Hartman, J. D., Bakos, G. Á., Kovács, G., \& Noyes, R. W. 2010, MNRAS, 408, 475

Hartman, J. D., Bakos, G. Á., Noyes, R. W., et al. 2011, AJ, 141, 166

Hewett, P. C., Irwin, M. J., Bunclark, P., et al. 1985, MNRAS, 213, 971

Horne, K. 1986, PASP, 98, 609
Irwin, J., Berta, Z. K., Burke, C. J., et al. 2011, ApJ, 727, 56

Irwin, J., Charbonneau, D., Berta, Z. K., et al. 2009b, ApJ, 701, 1436

Irwin, J., Charbonneau, D., Nutzman, P., \& Falco, E. 2009a, IAU Symp. 253, Transiting Planets, ed. F. Pont, D. Sasselov, \& M. Holman (Cambridge: Cambridge Univ. Press), 37

Irwin, J., Irwin, M., Aigrain, S., et al. 2007, MNRAS, 375, 1449

Johnson, D. R. H., \& Soderblom, D. R. 1987, AJ, 93, 864

Kirkpatrick, J. D., Henry, T. J., \& McCarthy, D. W., Jr. 1991, ApJS, 77, 417

Kraus, A. L., \& Hillenbrand, L. A. 2007, AJ, 134, 2340

Kraus, A. L., Tucker, R. A., Thompson, M. I., Craine, E. R., \& Hillenbrand, L. A. 2011, ApJ, 728, 48

Kwee, K. K., \& van Woerden, H. 1956, Bull. Astron. Inst. Neth., 12, 327

Lacy, C. H. 1977, ApJ, 218, 444

Landolt, A. U. 1992, AJ, 104, 340

Leggett, S. K. 1992, ApJS, 82, 351

Leggett, S. K., Allard, F., Berriman, G., Dahn, C. C., \& Hauschildt, P. H. 1996, ApJS, 104, 117

Lépine, S., \& Shara, M. M. 2005, AJ, 129, 1483

Lopez-Morales, M. 2004, PhD thesis, Univ. North Carolina at Chapel Hill

López-Morales, M. 2007, ApJ, 660, 732

López-Morales, M., \& Ribas, I. 2005, ApJ, 631, 1120

Lucy, L. B. 1967, Z. Astrophys., 65, 89

Luhman, K. L. 1999, ApJ, 525, 466

Luhman, K. L., \& Rieke, G. H. 1998, ApJ, 497, 354

MacDonald, J., \& Mullan, D. J. 2011, ApJ, submitted (arXiv:1106.1452)

Metcalfe, T. S., Mathieu, R. D., Latham, D. W., \& Torres, G. 1996, ApJ, 456, 356

Morales, J. C., Gallardo, J., Ribas, I., et al. 2010, ApJ, 718, 502

Morales, J. C., Ribas, I., Jordi, C., et al. 2009, ApJ, 691, 1400

Mullan, D. J., \& MacDonald, J. 2001, ApJ, 559, 353

Nelson, B., \& Davis, W. D. 1972, ApJ, 174, 617

Nutzman, P., \& Charbonneau, D. 2008, PASP, 120, 317

O’Neal, D., Neff, J. E., Saar, S. H., \& Cuntz, M. 2004, AJ, 128, 1802

Popper, D. M., \& Etzel, P. B. 1981, AJ, 86, 102

Prša, A., Batalha, N., Slawson, R. W., et al. 2011, AJ, 141, 83

Reid, I. N., Hawley, S. L., \& Gizis, J. E. 1995, AJ, 110, 1838

Ribas, I. 2003, A\&A, 398, 239

Ribas, I. 2006, Ap\&SS, 304, 89

Rockenfeller, B., Bailer-Jones, C. A. L., \& Mundt, R. 2006, A\&A, 448, 1111

Rodono, M., Cutispoto, G., Pazzani, V., et al. 1986, A\&A, 165, 135

Shkolnik, E. L., Hebb, L., Liu, M. C., Reid, I. N., \& Collier Cameron, A. 2010, ApJ, 716, 1522

Southworth, J., Maxted, P. F. L., \& Smalley, B. 2004a, MNRAS, 351, 1277

Southworth, J., Zucker, S., Maxted, P. F. L., \& Smalley, B. 2004b, MNRAS, 355,986

Tegmark, M., Strauss, M. A., Blanton, M. R., et al. 2004, Phys. Rev. D, 69, 103501

Terndrup, D. M., Stauffer, J. R., Pinsonneault, M. H., et al. 2000, AJ, 119, 1303

Tody, D. 1993, in ASP Conf. Ser. 52, Astronomical Data Analysis Software and Systems II, ed. R. J. Hanisch, R. J. V. Brissenden, \& J. Barnes (San Francisco, CA: ASP), 173

Torres, G. 2007, ApJ, 671, L65

Torres, G. 2010, AJ, 140, 1158

Torres, G., Andersen, J., \& Giménez, A. 2010, A\&AR, 18, 67

Torres, G., \& Ribas, I. 2002, ApJ, 567, 1140

Vaccaro, T. R., Rudkin, M., Kawka, A., et al. 2007, ApJ, 661, 1112

van Hamme, W. 1993, AJ, 106, 2096

Viti, S., Jones, H. R. A., Maxted, P., \& Tennyson, J. 2002, MNRAS, 329, 290

Viti, S., Jones, H. R. A., Schweitzer, A., et al. 1997, MNRAS, 291, 780

West, A. A., Hawley, S. L., Bochanski, J. J., et al. 2008, AJ, 135, 785

West, A. A., Walkowicz, L. M., \& Hawley, S. L. 2005, PASP, 117, 706

Wilson, R. E., \& Devinney, E. J. 1971, ApJ, 166, 605

Woolf, V. M., \& Wallerstein, G. 2006, PASP, 118, 218

Zahn, J. 1977, A\&A, 57, 383

Zucker, S., \& Mazeh, T. 1994, ApJ, 420, 806 